\title{
RELATIVE KNOTS AND TANGENTIAL EQUIVALENCES
}

\author{
BY \\ MAX K. AGOSTON( $\left.{ }^{1}\right)$
}

Levine has studied the groups, $\theta^{m, n}$, of knotted homotopy $n$-spheres in $S^{m}$. These groups can be interpreted as $h$-cobordism classes of certain ball pairs $\left(D^{m}, D^{n}\right)$. In this paper we define a relativized version, $\theta^{m, n}\left(V^{k}\right)$, of these groups, where $V^{k}$ is a $k$-manifold. $\theta^{m, n}(V)$ is the group of $h$-cobordism classes of pairs $\left(V \times D^{m}, V \times D^{n}\right)$, although in practice we find it convenient to give the formal definition in terms of maps rather than manifold pairs. Of course, $\theta^{m, n}$ (point) $=\theta^{m, n}$ as a special case. We shall show that the groups $\theta^{m, n}(V)$ and their framed analogues $\theta_{F}^{m, n}(V)$ fit into exact sequences (see $\S 3$ ) generalizing those of [11] and [4]. Finally, we use these groups to obtain necessary and sufficient conditions under which a "partial" stable tangential homotopy equivalence $f: M^{n} \rightarrow W^{n}$ (i.e., $f \mid[n / 2]-$ skeleton is tangential) is actually a stable tangential homotopy equivalence (see $\$ 9$ ). We rely heavily on the techniques of [11] and [10]. The author would like to thank Professor R. H. Szczarba for his encouragement and help.

0 . Notation. Throughout this paper all manifolds will be $C^{\infty}$, compact, and oriented. All maps are transverse to any boundaries and corners shall not worry us. If $M^{n}$ is a submanifold of $W^{m}$, we orient the normal bundle $\nu$ of $M$ in $W$ by the equation

$$
\tau_{M}+\nu=\tau_{W} \mid M,
$$

where $\tau_{M}, \tau_{W}$ are the tangent bundles of $M$ and $W$. The boundary of $M, \partial M$, is oriented by the equation

$$
\xi+\tau_{\partial M}=\tau_{M} \mid \partial M,
$$

where $\xi$ is a line bundle oriented positively by the vector pointing out from $M$. $-M$ denotes $M$ with the negative orientation.

$\boldsymbol{R}^{n}$ will denote $n$-dimensional real vector space with unit cube $I^{n}$ and unit disc $D^{n}$ - all with their natural orientations. Let $S^{n-1} \equiv \partial D^{n}, J^{n-1} \equiv \partial I^{n}-I^{n-1}$, where int $X$ or $\dot{X}$ denotes the interior of $X$. Let $\mathrm{Cl}(X)$ be the closure of $X . Z$ and $Z_{l}$ will mean the integers and cyclic group of order $l$, respectively.

1. Definition of $\theta^{m, n}\left(V^{k}\right), \theta_{F}^{m, n}\left(V^{k}\right)$. Let $V^{k}$ be a closed manifold. In this section we define the sets $\theta^{m, n}\left(V^{k}\right)$ and $\theta_{F}^{m, n}\left(V^{k}\right)$ and show how one can introduce a group

Received by the editors June 12, 1967.

(1) This research was partially supported by a National Science Foundation Fellowship and is part of the author's thesis submitted to Yale University. 
structure in them which is abelian if $n \geqq 2$. We set $q \equiv m-n$ throughout this paper.

Let $n \geqq 0$.

DefinITION. A map $f: V \times I^{n} \rightarrow V \times I^{n} \times D^{q}$ is called admissible if

(1) $f$ is an imbedding,

(2) $f(x, t)=(x, t, 0)$, for $t \in J^{n-1}$,

(3) $f\left(V \times \partial I^{n}\right)=V \times \partial I^{n} \times 0$,

(4) $f\left(V \times I^{n}\right) \subseteq \operatorname{int}\left(V \times I^{n} \times D^{q}\right)$.

Suppose that $f, g: V \times I^{n} \rightarrow V \times I^{n} \times D^{q}$ are admissible.

Definition. A map $H: V \times I^{n} \times I \rightarrow V \times I^{n} \times D^{q} \times I$ is said to connect $f$ and $g$, or $f \sim_{H} g$, if

(1) $H$ is an imbedding

(2) $H(x, t, 0)=(f(x, t), 0), H(x, t, 1)=(g(x, t), 1)$,

(3) $H(x, t, s)=(x, t, 0, s)$, for $t \in J^{n-1}$,

(4) $H\left(V \times \partial I^{n} \times I\right)=V \times \partial I^{n} \times 0 \times I$,

(5) $H$ (int $\left.\left(V \times I^{n} \times I\right)\right) \subseteq$ int $\left(V \times I^{n} \times D^{q} \times I\right)$.

It is easy to see that defining $f \sim g$ if there exists an $H$ such that $f \sim_{H} g$, makes $\sim$ into an equivalence relation in the set of all admissible maps.

Definition. $\theta^{m, n}\left(V^{k}\right)$ is the set of equivalence classes of admissible maps $f: V \times I^{n} \rightarrow V \times I^{n} \times D^{q}$ with respect to the relation $\sim$.

Next we define $\theta_{F}^{m, n}\left(V^{k}\right)$, the framed analogue of $\theta^{m, n}\left(V^{k}\right)$.

Definition. Let $\mathscr{F}: V \times I^{n} \times D^{q} \rightarrow V \times I^{n} \times D^{q}$ be a map. Let $f: V \times I^{n} \rightarrow$ $V \times I^{n} \times D^{q}$ be defined by $f(x, t)=\mathscr{F}(x, t, 0) .(f, \mathscr{F})$ is called $F$-admissible if

(1) $\mathscr{F}$ is a diffeomorphism,

(2) $f$ is admissible,

(3) $\mathscr{F}(x, t, y)=\left(p_{1} f(x, t), p_{2} f(x, t), y\right)$, for $t \in \partial I^{n}$, where $p_{i}$ will always denote the natural projection onto the $i$ th factor.

Suppose that $(f, \mathscr{F})$ and $(g, \mathscr{G})$ are $F$-admissible, with $\mathscr{F}, \mathscr{G}: V \times I^{n} \times D^{q}$ $\rightarrow V \times I^{n} \times D^{q}$.

DEFINITION. Let $\mathscr{H}: V \times I^{n} \times D^{q} \times I \rightarrow V \times I^{n} \times D^{q} \times I$ be a map and define $H: V \times I^{n} \times I \rightarrow V \times I^{n} \times D^{q} \times I$ by $H(x, t, s)=\mathscr{H}(x, t, 0, s) .(H, \mathscr{H})$ is said to F-connect $(f, \mathscr{F})$ and $(g, \mathscr{G})$, or $(f, \mathscr{F}) \sim_{(H, \mathscr{H}}(g, \mathscr{G})$, if

(1) $\mathscr{H}$ is a diffeomorphism,

(2) $\mathscr{H}(x, t, y, 0)=(\mathscr{F}(x, t, y), 0), \mathscr{H}(x, t, y, 1)=(\mathscr{G}(x, t, y), 1)$,

(3) $H$ connects $f$ and $g$,

(4) $\mathscr{H}(x, t, y, s)=\left(p_{1} H(x, t, s), p_{2} H(x, t, s), y, p_{4} H(x, t, s)\right)$, for $t \in \partial I^{n}$.

Let us define $(f, \mathscr{F}) \sim(g, \mathscr{G})$ if there is an $(H, \mathscr{H})$ so that $(f, \mathscr{F}) \sim_{(H, \mathscr{H})}(g, \mathscr{G})$. It is again clear that $\sim$ is an equivalence relation in the set of all $F$-admissible maps.

Definition. $\theta_{F}^{m, n}(V)$ is the set of equivalence classes of $F$-admissible maps $(f, \mathscr{F}), \mathscr{F}: V \times I^{n} \times D^{q} \rightarrow V \times I^{n} \times D^{q}$, with respect to the relation $\sim$.

In order to define products in $\theta^{m, n}(V)$ and $\theta_{F}^{m, n}(V)$ we need the following easy technical lemma which enables us to pick particularly nice representatives for each equivalence class. The proof will be omitted. 
We first introduce the following notation:

$$
\begin{aligned}
& I_{+}^{n} \equiv\left\{\left(t_{1}, \ldots, t_{n}\right) \in I^{n} \mid 0 \leqq t_{i} \leqq \frac{1}{2}, 1 \leqq i \leqq n\right\}, \\
& I_{-}^{n} \equiv\left\{\left(t_{1}, \ldots, t_{n}\right) \in I^{n} \mid \text { (a) if } n=1 \text {, then } \frac{1}{2} \leqq t_{1} \leqq 1\right. \text {, } \\
& \text { (b) if } n>1 \text {, then } \frac{1}{2} \leqq t_{i} \leqq 1 \text {, } \\
& \text { for } \left.1 \leqq i \leqq n-1 \text {, and } 0 \leqq t_{n} \leqq \frac{1}{2}\right\} \text {, }
\end{aligned}
$$

\section{LEMMA 1.1. Let $n \geqq 1$.}

(a) Let $u \in \theta^{m, n}(V)$. Then $u=\left[f_{+}\right]=\left[f_{-}\right]$, where $f_{ \pm}(x, t)=\left(p_{1} f\left(x, a_{ \pm}\right), t, 0\right)$, for $t \in I_{ \pm}^{n^{*}}$, and $f_{ \pm}\left(V \times I_{ \pm}^{n}\right) \subseteq \operatorname{int}\left(V \times I_{ \pm}^{n} \times D^{q}\right)$. Furthermore, if $f_{ \pm}^{\prime}$ satisfy the same conditions as $f_{ \pm}$, then there are connections $H_{ \pm}$between $f_{ \pm}^{\prime}$ and $f_{ \pm}$satisfying

$$
H_{ \pm}(x, t, s)=\left(p_{1} H_{ \pm}\left(x, a_{ \pm}, s\right), t, 0, p_{4} H_{ \pm}\left(x, a_{ \pm}, s\right)\right), \text { for } t \in I_{ \pm}^{n^{*}},
$$

and

$$
H_{ \pm}\left(\operatorname{int}\left(V \times I_{ \pm}^{n} \times I\right)\right) \subseteq \operatorname{int}\left(V \times I_{ \pm}^{n} \times D^{q} \times I\right) .
$$

(b) Let $v \in \theta_{F}^{m, n}(V)$. Then $v=\left[f_{+}, \mathscr{F}_{+}\right]=\left[f_{-}, \mathscr{F}_{-}\right]$, where $f_{ \pm}$satisfy the conditions in part (a) and $\mathscr{F}_{ \pm}(x, t, y)=\left(p_{1} f\left(x, a_{ \pm}\right), t, y\right)$, for $t \in I_{ \pm}^{n^{*}}$. Furthermore, if $\left(f_{ \pm}^{\prime}, \mathscr{F}_{ \pm}^{\prime}\right)$ satisfy the same conditions as $\left(f_{ \pm}, \mathscr{F}_{ \pm}\right)$, then there are F-connections $\left(H_{ \pm}, \mathscr{H}_{ \pm}\right)$ between $\left(f_{ \pm}^{\prime}, \mathscr{F}_{ \pm}^{\prime}\right)$ and $\left(f_{ \pm}, \mathscr{F}_{ \pm}\right)$with $H_{ \pm}$satisfying the conditions in part (a) and

$$
\mathscr{H}_{ \pm}(x, t, y, s)=\left(p_{1} H_{ \pm}\left(x, a_{ \pm}, s\right), t, y, p_{4} H_{ \pm}\left(x, a_{ \pm}, s\right)\right), \text { for } t \in I_{ \pm}^{n^{*}} .
$$

Using the notation of Lemma 1.1 we now define products for $n \geqq 1$ as follows:

Let $u, v \in \theta^{m, n}(V)$. Then $u=\left[f_{+}\right]$and $v=\left[g_{-}\right]$. Define $f_{+} * g_{-}: V \times I^{n} \rightarrow V \times I^{n}$ $\times D^{q}$ by

$$
\begin{aligned}
f_{+} * g_{-}(x, t) & =f_{+}\left(p_{1} g_{-}\left(x, a_{-}\right), t\right), & & \text { for } t \in I_{-}^{n^{*}}, \\
& =g_{-}(x, t), & & \text { for } t \in I_{+}^{n^{*}} .
\end{aligned}
$$

Define $u * v \equiv\left[f_{+} * g_{-}\right]$.

Let $u, v \in \theta_{F}^{m, n}(V)$. Then $u=\left[f_{+}, \mathscr{F}_{+}\right]$and $v=\left[g_{-}, \mathscr{G}_{-}\right]$. Define $u * v \equiv$ $\left[f_{+} * g_{-}, \mathscr{F}_{+} \mathscr{G}_{-}\right]$.

LEMMA 1.2. Let $n \geqq 1$.

(a) * is well defined in $\theta^{m, n}(V)$ and $\theta_{F}^{m, n}(V)$.

(b) $\left(\theta^{m, n}(V), *\right)$ and $\left(\theta_{F}^{m, n}(V), *\right)$ are groups which are abelian if $n \geqq 2$.

The proof of Lemma 1.2 will be deferred until $\S 4$. (If $[f, \mathscr{F}],[g, \mathscr{G}] \in \theta_{F}^{m .0}(V)$, set $[f, \mathscr{F}] *[g, \mathscr{G}] \equiv[\mathscr{F} g, \mathscr{F} \mathscr{G}]$. This defines a group structure also in $\theta_{F}^{m, 0}(V)$.)

REMARKS. If $n \geqq 2$, then the condition in Lemma 1.1 (a) reads $f_{ \pm}(x, t)=(x, t, 0)$, for $t \in I_{ \pm}^{n^{*}}$. There are corresponding simplifications in the rest of Lemma 1.1. As a matter of fact, the reason that this lemma and others to follow may seem more complicated than they really are is because we are trying to handle the cases $n=1$ and $n>1$ simultaneously. The reader is urged to separate the cases in order to 
clarify the situation. We also point out here that isotopies of admissible ( $F$ admissible) maps give rise to connections ( $F$-connections). This easy fact will be often used implicitly. Finally, we want to emphasize that defining the groups $\theta^{m . n}(V)$ in terms of maps and connections, is equivalent to defining them as cobordism classes of pairs $\left(V \times I^{n} \times D^{q}, V \times I^{n}\right)$ with suitable restrictions (similarly for $\left.\theta_{F}^{m, n}(V)\right)$; however, we found it simple to work with maps.

2. Generalized homotopy groups. In order to be able to state our main tileorems we need a "generalization" of the usual homotopy groups. For a detailed discussion of these groups see Yuh-lin Jou [16].

Let $Z$ be a fixed space (if $Z=\left\{z_{0}\right\}$, we will get the standard homotopy groups).

DEFINITION. Let $i \geqq 0$ and define

$\pi_{i}^{Z}\left(X, A, x_{0}\right) \equiv$ homotopy classes of naps $g: Z \times I^{i} \rightarrow X$ satisfying

(i) $g\left(Z \times J^{i-1}\right)=x_{0}$ and (ii) $g\left(Z \times I^{i-1}\right) \subseteq A$.

The product structure in $\pi_{i}^{Z}\left(X, A, x_{0}\right)$ is defined as follows for $i \geqq 2$ : Let $[f]$, $[g]$ $\in \pi_{i}^{Z}\left(X, A, x_{0}\right)$. Then $[f] *[g]=[h]$, where $h: Z \times I^{i} \rightarrow X$,

$$
\begin{array}{rlrl}
h\left(z,\left(t_{1}, \ldots, t_{i}\right)\right) & =f\left(z,\left(2 t_{1}, t_{2}, \ldots, t_{i}\right)\right), & & \text { for } 0 \leqq t_{1} \leqq \frac{1}{2} \\
& =g\left(z,\left(2 t_{1}-1, t_{2}, \ldots, t_{i}\right)\right), & \text { for } \frac{1}{2} \leqq t_{1} \leqq 1
\end{array}
$$

One can also define such a product in $\pi_{1}^{Z}\left(X, x_{0}\right)$.

Definition. Let $\left(X ; A, B, x_{0}\right)$ be a triad and define, for $i \geqq 2$, $\pi_{i}^{Z}\left(X ; A, B, x_{0}\right) \equiv$ homotopy classes of maps $g: Z \times I^{i} \rightarrow X$ satisfying

(i) $g\left(Z \times I^{i-1}\right) \subseteq B$, (ii) $g\left(Z \times J^{i-1}\right) \subseteq A$, and (iii) $g\left(Z \times J^{i-2} \times I\right)=x_{0}$. $\tilde{\pi}_{i}^{Z}\left(X ; A, B, x_{0}\right) \equiv$ homotopy classes of maps $g: Z \times I^{i} \rightarrow X$ satisfying

(i) $g\left(Z \times I^{i-1}\right) \subseteq B$, (ii) $g\left(Z \times J^{i-1}\right) \subseteq A$, and (iii) $g\left(Z \times \partial I^{i-1} \times I\right)=x_{0}$.

If $i \geqq 3$, then one can define a product just like in $\pi_{i}^{Z}\left(X, A, x_{0}\right)$. We shall drop base points from now on.

LEMMA 2.1. (a) $\pi_{i}^{Z}(X, A)$ is a group for $i \geqq 2$, which is abelian if $i \geqq 3 . \pi_{1}^{Z}(X)$ is also a group and $\pi_{2}^{Z}(X)$ is abelian.

(b) $\pi_{i}^{Z}(X ; A, B)$ and $\tilde{\pi}_{i}^{Z}(X ; A, B)$ are groups for $i \geqq 3$ which are abelian if $i \geqq 4$.

(c) The natural sequences

$$
\begin{aligned}
& \cdots \rightarrow \pi_{i}^{Z} A \rightarrow \pi_{i}^{Z} X \rightarrow \pi_{i}^{Z}(X, A) \rightarrow \pi_{i-1}^{Z} A \rightarrow \cdots \\
& \cdots \rightarrow \pi_{i}^{Z}(B, A \cap B) \rightarrow \pi_{i}^{Z}(X, A) \rightarrow \pi_{i}^{Z}(X ; A, B) \rightarrow \pi_{i-1}^{Z}(B, A \cap B) \rightarrow \cdots
\end{aligned}
$$

are exact.

Proof. It suffices to observe that the above groups are just the usual homotopy groups of certain function spaces, e.g., $\pi_{i}^{Z}\left(X, A, x_{0}\right) \approx \pi_{i}\left(X^{Z}, A^{Z}, x_{0}^{z}\right)$ and

$$
\pi_{i}^{Z}\left(X ; A, B, x_{0}\right) \approx \pi_{i}\left(X^{Z} ; A^{Z}, B^{Z}, x_{0}^{Z}\right) .
$$

If $X$ is an $H$-space, then one can define a monoid structure in the homotopy groups by pointwise multiplication. One can show without much trouble that this 
product agrees with the one just defined above. In fact, if $X$ is a topological group, then $\pi_{i}^{Z}\left(X, A, x_{0}\right)$ is a group for $i \geqq 0$; and $\pi_{i}^{Z}\left(X ; A, B, x_{0}\right), \tilde{\pi}_{i}^{Z}\left(X ; A, B, x_{0}\right)$ are groups for $i \geqq 2$. Next, let $G_{n}$ be the space of maps of $S^{n-1} \rightarrow S^{n-1}$ with degree 1 , and let $S O_{n}$ be the space of orthogonal maps in $G_{n}$. We want to point out for future reference that $\pi_{1}^{Z}\left(G_{q}, S O_{q}\right), \pi_{2}^{Z}\left(G_{q+l} ; S O_{q+l}, G_{q}\right)$, and $\tilde{\pi}_{2}^{Z}\left(G_{q+l} ; S O_{q+l}, G_{q}\right)$ are also groups.

3. The exact sequences. We are now ready to display the main results which generalize those of Levine [11] and Haefliger [4]. For the remainder of this paper assume that $V^{k}$ is a closed, 1-connected manifold. Define groups $P_{n}$ as follows:

$$
\begin{aligned}
P_{n} & =Z, & & n \equiv 0(4), \\
& =Z, & & n \equiv 2(4), \\
& =0, & & n \text { odd } .
\end{aligned}
$$

In $\S 6$ we shall define homomorphisms $\omega_{i}, \phi_{i}, \partial_{i}$ making the following sequences exact for $q \geqq 3, n \geqq 1, n+k \geqq 6$ :

$(1)_{q} \cdots \rightarrow \pi_{n}^{V} S O_{q} \stackrel{\omega_{1}}{\rightarrow} \theta_{F}^{m, n}(V) \stackrel{\phi_{1}}{\rightarrow} \theta^{m, n}(V) \stackrel{\partial_{1}}{\rightarrow} \pi_{n-1}^{V} S O_{q} \rightarrow \cdots$

$(2)_{q} \cdots \rightarrow \theta_{F}^{m, n}(V) \stackrel{\omega_{2}}{\longrightarrow} \pi_{n}^{V} G_{q} \stackrel{\phi_{2}}{\rightarrow} P_{k+n} \stackrel{\partial_{2}}{\longrightarrow} \theta_{F}^{m-1, n-1}(V) \stackrel{\omega_{2}}{\longrightarrow} \pi_{n-1}^{V} G_{q} \rightarrow \cdots$

(3) $)_{q} \cdots \rightarrow \theta^{m, n}(V) \stackrel{\omega_{3}}{\rightarrow} \pi_{n}^{V}\left(G_{q}, S O_{q}\right) \stackrel{\phi_{3}}{\rightarrow} P_{k+n} \stackrel{\partial_{3}}{\longrightarrow} \theta^{m-1, n-1}(V) \stackrel{\omega_{3}}{\rightarrow} \pi_{n-1}^{V}\left(G_{q}, S O_{q}\right) \rightarrow \cdots$

Let

$(4)_{q} \cdots \rightarrow \pi_{n}^{V} S O_{q} \stackrel{\omega_{4}}{\rightarrow} \pi_{n}^{V} G_{q} \stackrel{\phi_{4}}{\rightarrow} \pi_{n}^{V}\left(G_{q}, S O_{q}\right) \stackrel{\partial_{4}}{\rightarrow} \pi_{n-1}^{V} S O_{q} \rightarrow \cdots$ be the exact sequence of the pair $\left(G_{q}, S O_{q}\right)$. Then we have a commutative (up to sign) diagram:

$(5)_{q}$

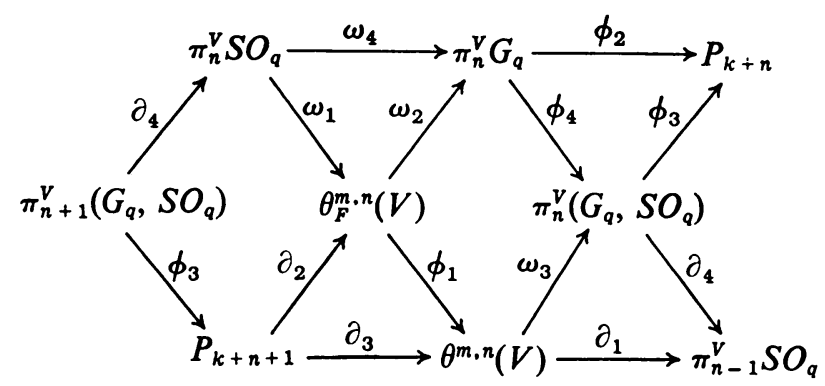

The commutativity (up to sign) of (5) $)_{q}$ and the exactness of $(1)_{q}-(3)_{q}$ is the content of Theorem 1. In $\S 8$ we discuss the suspension maps $s$ and define maps $\omega_{5}, \phi_{5}, \omega_{6}$, $\phi_{6}$ which make the following sequences exact (Theorem 2 and 3):

$(6)_{q} \cdots \rightarrow \pi_{n+1}^{V}\left(G_{q+l} ; S O_{q+l}, G_{q}\right) \stackrel{\omega_{5}}{\rightarrow} \theta^{m, n}(V) \stackrel{s}{\rightarrow} \theta^{m+l, n}(V) \stackrel{\phi_{5}}{\longrightarrow} \pi_{n}^{V}\left(G_{q+l} ; S O_{q+l}, G_{q}\right)$ $\stackrel{\omega_{5}}{\rightarrow} \theta^{m, n-1}(V) \rightarrow \cdots$

$(7)_{q} \cdots \rightarrow \tilde{\pi}_{n+1}^{V}\left(G_{q+l} ; S O_{q+l}, G_{q}\right) \stackrel{\omega_{6}}{\rightarrow} \theta_{F}^{m, n}(V) \stackrel{s}{\rightarrow} \theta_{F}^{m+l, n}(V) \stackrel{\Phi_{6}}{\rightarrow} \tilde{\pi}_{n}^{V}\left(G_{q+l} ; S O_{q+l}, G_{q}\right)$ $\stackrel{\omega_{B}}{\rightarrow} \theta_{F}^{m, n-1}(V) \rightarrow \cdots$.

4. Some preliminaries. We begin by proving Lemma 1.2.

Proof of Lemma 1.2. (a) This is a fairly straightforward exercise and we shall show only that $*$ is well defined in $\theta^{m, n}(V)$, since the proof for $\theta_{F}^{m, n}(V)$ is similar. 
First, observe that $f_{+} * g_{-}$is a well defined admissible map. Suppose that we had chosen $f_{+}^{\prime}, g_{-}^{\prime}$ instead of $f_{+}, g_{-}$respectively. Choose a connection $H_{ \pm}$as in Lemma 1.1(a). Define $H_{+} * H_{-}: V \times I^{n} \times I \rightarrow V \times I^{n} \times D^{q} \times I$ by

$$
\begin{aligned}
H_{+} * H_{-}(x, t, s) & =H_{+}\left(p_{1} H_{-}\left(x, a_{-}, s\right), t, p_{4} H_{-}\left(x, a_{-}, s\right)\right), & & \text { for } t \in I_{-}^{n^{*}}, \\
& =H_{-}(x, t, s), & & \text { for } t \in I_{+}^{n^{*}} .
\end{aligned}
$$

Then $H_{+} * H_{-}$is a connection between $f_{+} * g_{-}$and $f_{+}^{\prime} * g_{-}^{\prime}$. Therefore $*$ is well defined.

(b) Define $\mathscr{E}: V \times I^{n} \times D^{q} \rightarrow V \times I^{n} \times D^{q}, \varepsilon: V \times I^{n} \rightarrow V \times I^{n} \times D^{q}$ by $\mathscr{E}(x, t, y)$ $=(x, t, y), \varepsilon(x, t)=(x, t, 0)$. Then $[\varepsilon]$ and $[\varepsilon, \mathscr{E}]$ serve as identities for $*$ in $\theta^{m, n}(V)$ and $\theta_{F}^{m, n}(V)$, respectively. Associativity of $*$ follows from a more complicated version of Lemma 1.1 and it will be left to the doubting reader to fill in the somewhat lengthy details.

Suppose $n \geqq 3$. The following sequence of figures shows the isotopies required to prove that $*$ is commutative.

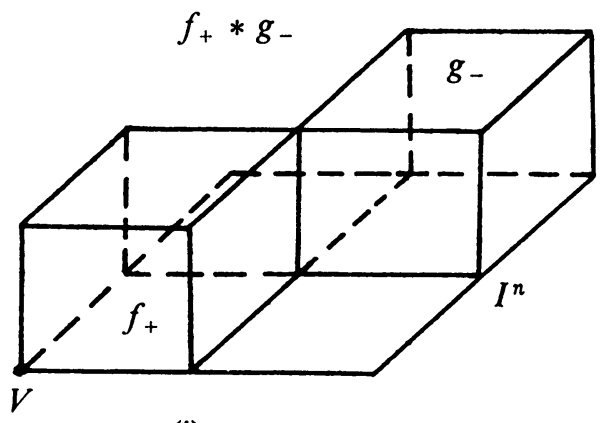

(i)

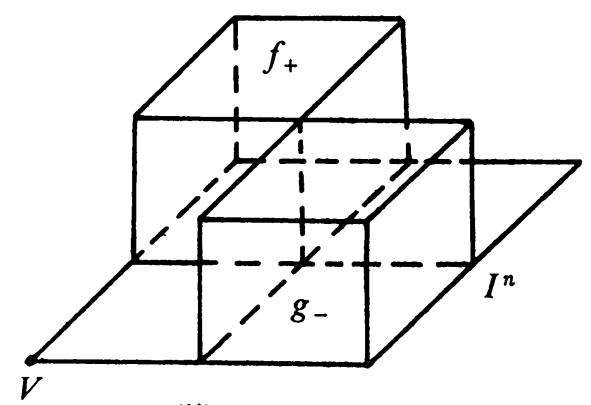

(ii)

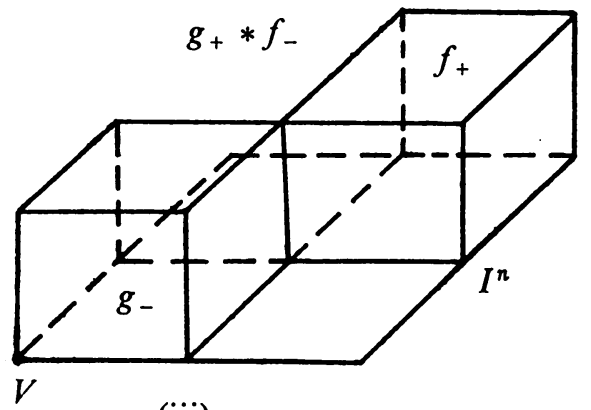

(iii)

Proving commutativity in case $n=2$ is slightly more technical but essentially the same.

Now let $[f] \in \theta^{m, n}(V), n \geqq 1$ and let $[f]=\left[f_{-}\right]$as usual. An inverse of $[f]$ is obtained by reflecting $f_{-}$about the plane $t_{1}=\frac{1}{2}$. If $n=1$, one must also compensate for the fact that $p_{1} f_{-}(, 0): V \rightarrow V$ is not necessarily the identity. Since the explicit 
construction for a representative $g$ of $[f]^{-1}$ is complicated but straightforward, it will be omitted.

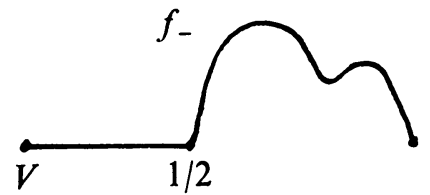

$V \times I^{n} \times D^{q}$

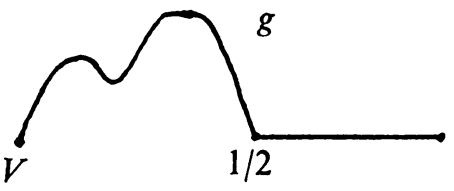

$V \times I^{n} \times D^{q}$

Next we define two constructions which will be used frequently and then conclude with some technical lemmas important for the subsequent development.

Definition. Let $h: X \times S^{l} \rightarrow Y \times S^{l}$ and

$$
h^{\prime}: X \rightarrow S^{l^{s l}}
$$

be maps (identify $S^{l}$ with $y \times S^{l}$ to take care of the case $h: X \times S^{l} \rightarrow S^{l}$ ). Then

$$
\underline{\sigma(h)}: X \rightarrow S^{\iota^{s l}}
$$

and

$$
\underline{\rho\left(h^{\prime}\right)}: X \times S^{l} \rightarrow S^{l}
$$

are defined by $\sigma(h)(x)(u)=p_{2} h(x, u)$ and $\rho\left(h^{\prime}\right)(x, u)=h^{\prime}(x)(u)$.

LEMMA 4.1. Let $q \geqq 3, n \geqq 1, m+k \geqq 6$.

(a) Let $[f] \in \theta^{m, n}(V)$. Then there is a diffeomorphism $\mathscr{F}^{0}: V \times I^{n} \times D^{q} \rightarrow V \times I^{n} \times D^{q}$ satisfying

(i) $\mathscr{F}^{0}(x, t, 0)=f(x, t)$,

(ii) $\mathscr{F}^{0}(x, t, y)=(x, t, y)$, for $t \in J^{n-1}$,

(iii) $\mathscr{F}^{0}(x, t, y)=\left(p_{1} f(x, t), p_{2} f(x, t), \alpha(x, t)(y)\right)$, for $t \in I^{n-1}$, where $\alpha: V \times I^{n-1}$ $\rightarrow \mathrm{SO}_{a}$,

(iv) $\sigma\left(\mathscr{F}^{0}\right)\left(V \times I^{n}\right) \subseteq G_{q}$.

(b) Suppose that $[f]=\left[f^{\prime}\right]$ and $\mathscr{F} 0^{\circ}$ is chosen with respect to $f^{\prime}$ as in part (a). Let $H$ connect $f$ and $f^{\prime}$. Then there is a diffeomorphism $\mathscr{H}^{0}: V \times I^{n} \times D^{q} \times I \rightarrow V \times I^{n}$ $\times D^{q} \times I$ satisfying

(i) $\mathscr{H}^{0}(x, t, 0, s)=H(x, t, s)$,

(ii) $\mathscr{H}^{0}(x, t, y, 0)=\left(\mathscr{F}^{0}(x, t, y), 0\right), \mathscr{H}^{0}(x, t, y, 1)=\left(\mathscr{F}^{0}(x, t, y), 1\right)$,

(iii) $\mathscr{H}^{0}(x, t, y, s)=(x, t, y, s)$, for $t \in J^{n-1}$,

(iv) $\mathscr{H}^{0}(x, t, y, s)=\left(p_{1} H(x, t, s), p_{2} H(x, t, s), \tilde{\alpha}(x, t, s)(y), p_{4} H(x, t, s)\right)$, for $t \in I^{n-1}$, where $\tilde{\alpha}: V \times I^{n-1} \times I \rightarrow S O_{q}$,

(v) $\sigma\left(p_{3} \mathscr{H}^{0} R\right)\left(V \times I^{n} \times I\right) \subseteq G_{q}$, where $R: V \times I^{n} \times I \times D^{q} \rightarrow V \times I^{n} \times D^{q} \times I$ is given by $R(x, t, s, y)=(x, t, y, s)$.

(c) Let $u \in \theta^{m, n}(V)$ and choose $f_{ \pm}$as in Lemma 1.1(a) so that $u=\left[f_{ \pm}\right]$. Then there are diffeomorphisms $\mathscr{F}_{ \pm}^{0}: V \times I^{n} \times D^{q} \rightarrow V \times I^{n} \times D^{q}$ satisfying (i)-(iv) of part (a) and also

(v) $\mathscr{F}_{ \pm}^{0}(x, t, y)=\left(p_{1} \mathscr{F}_{ \pm}^{0}\left(x, a_{ \pm}, y\right), t, p_{3^{\prime}}^{\mathscr{F}_{ \pm}^{0}}\left(x, a_{ \pm}, y\right)\right)$, for $t \in I_{ \pm}^{n^{*}}$. 
Proof. We shall content ourselves with outlining a proof only for part (a); everything is really straightforward. Since the normal bundle of $f$ is trivial one can get a trivialization $\mathscr{F}^{0}$ of a tubular neighborhood $T$ which we may assume to be all of $V \times I^{n} \times D^{a}$ by Smale theory [14, Theorem 4.1]. Furthermore, we could have let the trivialization be induced from the standard one on $V \times J^{n-1} \times 0$. If one also uses the uniqueness of tubular neighborhoods theorem on $V \times I^{n-1} \times 0$ one gets $\mathscr{F}^{0}$ to satisfy (i)-(iv).

REMARK. Lemma 4.1 says roughly that every admissible map almost comes from an $F$-admissible pair. $\left(f, \mathscr{F}^{0}\right)$ fails to be $F$-admissible only on $V \times I^{n-1} \times 0$.

Lemma 4.2. Let $q \geqq 3, n \geqq 0, m+k \geqq 6$. Let $[f, \mathscr{F}] \in \theta_{F}^{m, n}(V)$. If $[f]=[g] \in \theta^{m, n}(V)$, then $[f, \mathscr{F}]=[g, \mathscr{G}]$ for some $\mathscr{G}$.

Proof. The proof is similar to that of Lemma 4.1 and easy. If $H$ connects $f$ and $g$, choose a trivialization $\mathscr{H}$ of its normal bundle which is induced from $\mathscr{F}$. $\mathscr{G}$ will then be the restriction of $\mathscr{H}$ to $g$.

Lemma 4.3. Let $q \geqq 3, n \geqq 1, m+k \geqq 6$. Choose $\mathscr{F}^{0}, \mathscr{G}^{0}$ with respect to admissible maps $f, g$ as in Lemma 4.1(a). Then $\left[\sigma\left(\mathscr{F}^{0} \mathscr{G}^{0}\right)\right]=\left[\sigma\left(\mathscr{F}^{0}\right) \sigma\left(\mathscr{G}^{0}\right)\right] \in \pi_{n}^{V}\left(G_{q}, S O_{q}\right)$.

Proof. Let $\mathscr{F}_{+}^{0}, \mathscr{G}_{-}^{0}$ be chosen for $f, g$, respectively, as in Lemma 4.1(c). By Lemma 4.1(b) $\sigma\left(\mathscr{F}^{0}\right) \simeq \sigma\left(\mathscr{F}_{+}^{0}\right)$ and $\sigma\left(\mathscr{G}^{0}\right) \simeq \sigma\left(\mathscr{G}_{-}^{0}\right)$; and so $\sigma\left(\mathscr{F}^{0}\right) \sigma\left(\mathscr{G}^{0}\right) \simeq \sigma\left(\mathscr{F}_{+}^{0}\right) \sigma\left(\mathscr{G}_{+}^{0}\right)$. Since the connections between $\mathscr{F}^{0}, \mathscr{F}_{+}^{0}$ and $\mathscr{G}^{0}, \mathscr{G}_{-}^{0}$ could have been chosen to be level preserving we also have that $\sigma\left(\mathscr{F}^{0} \mathscr{G}^{0}\right) \simeq \sigma\left(\mathscr{F}_{+}^{0} \mathscr{G}_{-}^{0}\right)$. It suffices to show therefore that $\sigma\left(\mathscr{F}_{+}^{0} \mathscr{G}_{-}^{0}\right) \simeq \sigma\left(\mathscr{F}_{+}^{0}\right) \sigma\left(\mathscr{G}_{-}^{0}\right)$.

Now,

$$
\begin{aligned}
\sigma\left(\mathscr{F}_{+}^{0} \mathscr{G}_{-}^{0}\right)(x, t)(y) & =p_{3} \mathscr{G}_{-}^{0}(x, t, y), & & \text { for } t \in I_{+}^{n^{*}}, \\
& =p_{3} \mathscr{F}_{+}^{0}\left(p_{1} \mathscr{G}_{-}^{0}\left(x, a_{-}, y\right), t, p_{3} \mathscr{G}_{-}^{0}\left(x, a_{-}, y\right)\right), & & \text { for } t \in I_{-}^{n^{*}} . \\
\left(\sigma\left(\mathscr{F}_{+}^{0}\right) \sigma\left(\mathscr{G}_{-}^{0}\right)\right)(x, t)(y) & =p_{3} \mathscr{G}_{-}^{0}(x, t, y), & & \text { for } t \in I_{+}^{n^{*}} \\
& =p_{3} \mathscr{F}_{+}^{0}\left(x, t, p_{3} \mathscr{G}_{-}^{0}(x, t, y)\right), & & \text { for } t \in I_{-}^{n^{*}} .
\end{aligned}
$$

But $p_{1} \mathscr{G}_{-}^{0}\left(, a_{-}, y\right)=p_{1} g\left(, a_{-}\right): V \rightarrow V$ is homotopic to the identity and so we are done.

AdDENDUM to Lemma 4.3. If $(f, \mathscr{F})$ and $(g, \mathscr{G})$ are F-admissible, then $[\sigma(\mathscr{F} \mathscr{G})]$ $=[\sigma(\mathscr{F}) \sigma(\mathscr{G})] \in \pi_{n}^{V} G_{q}$.

Proof. Same as for Lemma 4.3. One merely needs to observe that all the appropriate homotopies may be taken with respect to $\pi_{n}^{V} G_{q}$. By using Lemma 1.1(b) instead of Lemma 4.1 we only need $n \geqq 1$.

Before stating the next lemma we must make a few remarks about the Thom map. By $\left(W^{n}, \mathscr{G}\right) \subseteq{ }^{g} M^{m}$ we shall mean imbeddings $g: W \rightarrow M, \mathscr{G}: W \times D^{m-n} \rightarrow M$ so that $\mathscr{G}(x, 0)=g(x) . \mathscr{G}$ will be called a framing of $W$. Recall that given $\left(W^{n}, \mathscr{G}\right)$ $\subseteq^{g} M^{m}$ we can define a map $t(W, \mathscr{G}): M \rightarrow S^{m-n}$ called the Thom map (for an explicit definition see $\left[7\right.$, p. 346]). In particular, given $\left(W^{k+n}, \mathscr{G}\right) \subseteq{ }^{g} V \times I^{n} \times S^{q-1}$ 
we obtain a map $t(W, \mathscr{G}): V \times I^{n} \times S^{q-1} \rightarrow S^{q-1}$. When does $\sigma(t(W, \mathscr{G}))$ determine an element of $\pi_{n}^{V} G_{q}$ ? The case $n=0$ is essentially handled by [11, Lemma 3.6], i.e., the inclusion $W \rightarrow V \times S^{q-1}$ followed by the projection $V \times S^{q-1} \rightarrow V$ must define a map $W \rightarrow V$ of degree 1 . Suppose $n \geqq 1$, and let $e \equiv(0, \ldots, 0,1)$. If $\partial W=$ $W \cap\left(V \times \partial I^{n} \times S^{q-1}\right)=V \times \partial I^{n} \times e$ and $\mathscr{G} \mid \partial W$ is the standard framing of $V \times \partial I^{n} \times e$ in $V \times \partial I^{n} \times S^{q-1}$, then one can also easily see that $\sigma(t(W, \mathscr{G}))$ determines an element of $\pi_{n}^{V} G_{q}$. Since this will always be the case whenever we use the Thom map in such a context, we write simply $[t(W, \mathscr{G})] \in \pi_{n}^{V} G_{q}$ and shall not explicitly justify it in the future. (See [11, §1.3-1.5] for a more detailed discussion.)

Now define $D_{+}^{q} \equiv\left\{\left(t_{1}, \ldots, t_{q}\right) \in S^{q-1} \mid t_{q} \geqq 0\right\}$ and $D_{-}^{q} \equiv\left\{\left(t_{1}, \ldots, t_{q}\right) \in S^{q-1} \mid t_{q} \leqq 0\right\}$.

Lemma 4.4. Let $(f, \mathscr{F})$ be F-admissible and let $W^{k+n} \equiv \mathscr{F}\left(V \times I^{n} \times e\right)$. Define $\mathscr{G}: W^{k+n} \times D_{+}^{q-1} \rightarrow V \times I^{n} \times S^{q-1}$ by $\mathscr{G}(\mathscr{F}(x, t, e), y)=\mathscr{F}(x, t, y)$. Then

$$
\left[t\left(W^{k+n}, \mathscr{G}\right)\right] *[\sigma(\mathscr{F})]=0 \in \pi_{n}^{V} G_{q}, \text { for } n \geqq 1 .
$$

Proof. Clearly $\left[t\left(W^{k+n}, \mathscr{G}\right)\right]=[\sigma(\mathscr{F}-1)]$ and therefore $\left[t\left(W^{k+n}, \mathscr{G}\right)\right] *[\sigma(\mathscr{F})]$ $\left.=\left[\sigma^{-1}\right)\right] *[\sigma(\mathscr{F})]=\left[\sigma\left(\mathscr{F}^{-1}\right) \sigma(\mathscr{F})\right]=[\sigma(\mathscr{F}-1 \mathscr{F})]=0$.

The next lemma tells us when submanifolds of $V \times I^{n} \times D^{q}$ can be realized by admissible maps.

LEMMA 4.5. Let $W^{k+n}$ be a submanifold of $V \times I^{n} \times D^{q}, n \geqq 0$, satisfying

(1) $\partial W \cap\left(V \times J^{n-1} \times D^{q}\right)=V \times J^{n-1} \times 0$,

(2) $\partial W-\left(V \times J^{n-1} \times 0\right) \subseteq \operatorname{int}\left(V \times I^{n-1} \times D^{q}\right)$,

(3) int $W \subseteq$ int $\left(V \times I^{n} \times D^{q}\right)$,

(4) $W \leftarrow \tilde{H} V \times I^{n}, H\left(V \times J^{n-1}\right)=V \times J^{n-1} \times 0$.

Then there is an admissible map $f$ with $f\left(V \times I^{n}\right)=W$. Furthermore $[f] \in \theta^{m, n}(V)$ is uniquely determined by $W$ if $k+n \geqq 6$.

Proof. Let $F$ denote the composite

$$
V \times J^{n-1} \underset{H}{\longrightarrow} W \stackrel{\left(p_{1}, p_{2}\right)}{\longrightarrow} V \times J^{n-1} .
$$

$F$ obviously extends to a diffeomorphism $\tilde{F}$ of $V \times I^{n}$. Define $f \equiv H \tilde{F}^{-1}$. Then $f$ is admissible and $f\left(V \times I^{n}\right)=W$. Suppose that $f^{\prime}$ is admissible and $f^{\prime}\left(V \times I^{n}\right)=W$. Define

$$
K: V \times I^{n} \times\{0,1\} \cup V \times J^{n-1} \times I \rightarrow W \times I
$$

by $K(x, y, 0)=(f(x, t), 0), K(x, t, 1)=\left(f^{\prime}(x, t), 1\right)$, and $K(x, t, s)=(f(x, t), s)$, for $t \in J^{n-1}$. Using the techniques of [14] one can obtain a diffeomorphism $\tilde{K}: V \times I^{n} \times I$ $\rightarrow W \times I$ which extends $K$. But $\tilde{K}$ is a connection between $f$ and $f^{\prime}$. Thus $[f]=\left[f^{\prime}\right]$.

5. Some surgery. Suppose that $[g] \in \pi_{n}^{V}\left(G_{q}, S O_{q}\right)$. Let $I_{1}^{n-1} \equiv I^{n-1} \times 1 \times e$, and let $P: V \times I^{n} \times S^{q} \rightarrow V \times I_{1}^{n-1}$ be the natural projection. We may assume that $W^{k+n} \equiv \rho(g)^{-1}(e)$ is a manifold with framing $\mathscr{F}$ and that $\partial W^{k+n}=\left(V \times J^{n-1} \times e\right)$ $\cup M^{k+n-1}$, where $M \approx V \times I^{n-1}$ with the natural inclusion $V \times a_{+} \times e \rightarrow M$ a 
homotopy equivalence. (In our case here $P \mid M$ is actually a diffeomorphism, but the weaker assumption will be needed later on.) Let $r \equiv P \mid W, q \geqq 3, n \geqq 1$, and $k+n \geqq 6$.

LEMMA 5.1. We may suppose that $r$ is $([(k+n) / 2]-1)$-connected, i.e., $r_{\#}: \pi_{j} W$ $\rightarrow \pi_{j}\left(V \times I_{1}^{n-1}\right)$ is an isomorphism for $j \leqq[(k+n) / 2]-1$, where $[x]$ denotes the greatest integer in $x$.

Proof. The proof of this lemma and of others to follow will consist merely of reducing the situation to one handled elsewhere. For simplicity we make the following definitions: $N \equiv V \times I^{n} \times S^{q-1}, N_{1} \equiv V \times J^{n-1} \times S^{q-1}, T \equiv \mathscr{F}\left(W \times D^{q-1}\right)$, where, without loss of generality, $T \cap V \times J^{n-1} \times S^{q-1}=V \times J^{n-1} \times D_{+}^{q-1}$ and $T \cap V \times I^{n-1} \times S^{q-1} \subseteq V \times I^{n-1} \times S^{q-1}$,

$$
V_{1} \equiv \mathrm{Cl}\left[\left(V \times I^{n-1} \times S^{q-1}\right)-\left(T \cap\left(V \times I^{n-1} \times S^{q-1}\right)\right)\right] \approx V \times I^{n-1} \times D^{q-1},
$$

$V_{2} \equiv V \times J^{n-1} \times D_{+}^{q-1}, \quad V_{3} \equiv \mathrm{Cl}\left(\partial W-\left(V \times I_{1}^{n-1}\right)\right), \quad W_{1} \equiv \mathscr{F}(W \times e)$, and $\bar{r}: N-W$ $\rightarrow V_{1}, \bar{r}(x, t, y)=\mathscr{F}((x, 0, g(x, 0) e), e)$.

An argument like that in $[11, \S 4.9]$ shows that we may suppose that $N-W, r$, and $\bar{r}$ are 1-connected. Assume inductively that $r$ and $\bar{r}$ are $(s-1)$-connected for $2 \leqq s \leqq[(k+n) / 2]-1$. We want to make them $s$-connected.

Consider the split exact sequences

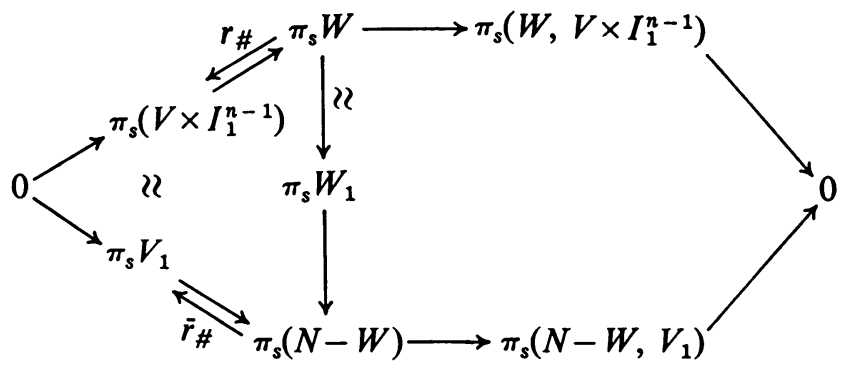

If $\bar{r}_{\#}$ is an isomorphism and if $[f] \in \pi_{s} W$, then $r_{\#}([f])=0$ implies that $\mathscr{F}(f \times e) \simeq 0$ in $N-W$. The techniques of [3], [11], and [9] now apply to make $r$-connected. Therefore, it suffices to show that $\bar{r}$ is $s$-connected; in fact, using the Whitehead theorem, it suffices to show that $H_{s}\left(N-W, V_{1}\right)=0$ (all homology and cohomology will be with integer coefficients), because we also have the split exact sequence

$$
0 \rightarrow H_{s} V_{1} \rightarrow H_{s}(N-W) \rightarrow H_{s}\left(N-W, V_{1}\right) \rightarrow 0 .
$$

Since

$$
\begin{aligned}
H_{s}\left(N-W, V_{1}\right) \approx H_{s}\left(\mathrm{Cl}(N-T), V_{1}\right) & \approx H^{k+m-s-1}\left(\mathrm{Cl}(N-T), \partial(\mathrm{Cl}(N-T))-V_{1}\right) \\
& \approx H^{k+m-s-1}\left(N, T \cup N_{1}\right),
\end{aligned}
$$

let us consider the exact sequence of the triple $\left(N, T \cup N_{1}, N_{1}\right)$ :

$$
\begin{aligned}
\cdots & \rightarrow H^{k+m-s-2}\left(T \cup N_{1}, N_{1}\right) \rightarrow H^{k+m-s-1}\left(N, T \cup N_{1}\right) \\
& \rightarrow H^{k+m-s-1}\left(N, N_{1}\right) \rightarrow H^{k+m-s-1}\left(T \cup N_{1}, N_{1}\right) \rightarrow \cdots
\end{aligned}
$$


Now $H^{*}\left(N, N_{1}\right)=0$; also,

$$
\begin{aligned}
H^{k+m-s-t}\left(T \cup N_{1}, N_{1}\right) & \approx H^{k+m-s-t}\left(T, V_{2}\right) \approx H^{k+m-s-t}\left(W, V \times I_{1}^{n-1}\right) \\
& \approx H_{s-q+t}\left(W, V_{3}\right)=0
\end{aligned}
$$

if $t \leqq 2$, because $q \geqq 3$ and $r$ is $(s-1)$-connected. Therefore $H^{k+m-s-1}\left(N, T \cup N_{1}\right)=0$ and we are done.

From now on assume that $r$ is $([(k+n) / 2]-1)$-connected. The proof of Lemma 5.1 shows that then $\bar{r}$ is $[(k+n) / 2]$-connected.

Lemma 5.2. Let $k+n=2 l$. Then $W \approx V^{k} \times I^{n} \pm U^{k+n}$, where $U$ is a r-manifold with boundary a homotopy sphere and \pm denotes boundary connected sum (see $[3, \S 1.2])$.

Proof. Let $r^{\prime}$ denote the natural projection $W \rightarrow V \times a_{+} \times e \subseteq M$. We have a split exact sequence

$$
0 \longrightarrow H_{i} M \stackrel{r_{*}^{\prime}}{\longleftrightarrow} H_{i} W \longrightarrow H_{i}(W, M) \longrightarrow 0 .
$$

Therefore $H_{i} W \approx H_{i} M \oplus H_{i}(W, M)$. Since $H_{i} M \approx H_{i} V^{\prime}$ and $H_{j} W \approx H_{j} V$ for $j<l$ (because $r$ is $(l-1)$-connected), we see immediately that $r^{\prime}$ is $(l-1)$-connected. We can now use the proof of [10, Proposition 5] to get our result, because $r$ is a normal map, i.e., it preserves the stable normal bundle.

LEMMA 5.3. $\gamma(U) \in P_{k+n}$ depends only on the homotopy class of $g$, where $\gamma$ is the function defined in $[11, \S 4.5]$.

Proof. Let $[g]=\left[g^{\prime}\right] \in \pi_{n}^{V}\left(G_{q}, S O_{q}\right)$, and let $h: V \times I^{n} \times I \rightarrow G_{q}$ be a homotopy between $g$ and $g^{\prime}$, i.e., $h\left|V \times I^{n} \times 0=g, h\right| V \times I^{n} \times 1=g^{\prime}, h\left(V \times I^{n-1} \times I\right) \subseteq S O_{q}$, $h\left(V \times J^{n-1} \times I\right)=$ identity. We may assume that $X^{k+n+1} \equiv R^{-1} \rho(h)^{-1}(e)$ is a manifold with framing $\mathscr{H}$ which is a framed cobordism between $(W, \mathscr{F})$ and $\left(-W^{\prime}, \mathscr{F}^{\prime}\right)$, where $\left(W^{\prime}, \mathscr{F}^{\prime}\right)$ is defined with respect to $g^{\prime}$ similarly to $(W, \mathscr{F}) .(R$ is defined as in Lemma 4.1. b.v.) Let $U^{k+n^{\prime}}$ be obtained similarly to $U$. We want to show that $\gamma(U)=\gamma\left(U^{\prime}\right)$.

Let $Y \equiv \partial\left(V \times I^{n} \times e \times I\right)$ and let $\tilde{r}: X \rightarrow V \times I^{n} \times e \times I$ be the restriction of the natural projection $V \times I \times S^{q-1} \times I \rightarrow V \times I^{n} \times e \times I$. By an argument similar to that in Lemma 5.1, we may assume that $\tilde{r}$ is $(l-1)$-connected. Let $\mu$ denote the fundamental class of a manifold and consider the diagram:

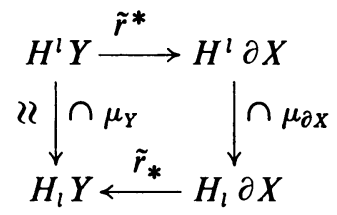

Therefore $H^{l} \partial X=\operatorname{Im} \tilde{r} \oplus A$, where $A \equiv\left(\bigcap \mu_{\partial X}\right)^{-1}\left(\operatorname{ker} \tilde{r}_{*}\right)$. Also, $\operatorname{Im} \tilde{r}^{*} \approx H^{l} Y$ since $\tilde{r}^{*}$ is $1-1$ by [10, Lemma 3]. 
Next, we look at the Mayer-Vietoris sequences:

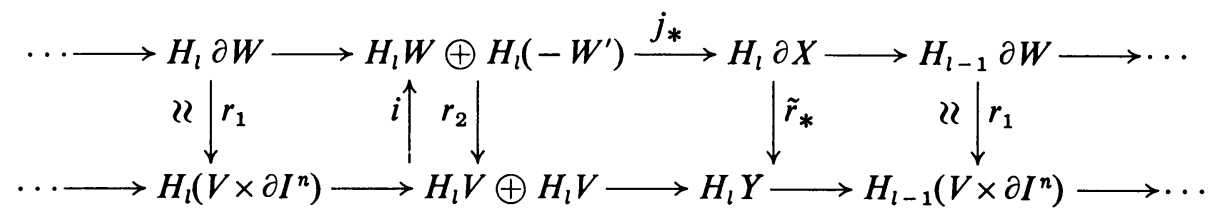

(We used the fact that $\partial X-\left(W \cup-W^{\prime}\right) \approx \partial W \times I$ and made other obvious identifications. $r_{1}$ and $r_{2}$ are the maps induced from $\tilde{r} . i$ is induced from the inclusions $V \subseteq W, W^{\prime}$, and $r_{2} i=1$.) Clearly ker $r_{2}=H_{l} U \oplus H_{l}\left(-U^{\prime}\right)$ and a little diagram chasing shows that $j_{*}$ is $1-1$ on $\operatorname{ker} r_{2}$ and $\operatorname{ker} \tilde{r}_{*}=j_{*}\left(\operatorname{ker} r_{2}\right)$.

Case (a) $l=2 s, k+n=4 s:\left[10\right.$, p. 26] shows that $\operatorname{Im} \tilde{r}^{*} \cup A=0$. Thus index $\partial X=\operatorname{index}\left(\operatorname{Im} \tilde{r}^{*}\right)+\operatorname{index} A$. But index $\partial X=0=$ index $Y$, since $\partial X$ and $Y$ bound. Also, index $\operatorname{Im} \tilde{r}^{*}=\operatorname{index} Y$; so index $A=0$. Finally, $H^{l}\left(\partial X, W^{\prime}\right) \cup H^{l}(\partial X, W)$ $\subseteq H^{2 l}(\partial X, \partial X)=0$ shows that $H^{l} U \cup H^{\prime}\left(-U^{\prime}\right)=0$, and so $0=\operatorname{index} A=\operatorname{index} U$ + index $\left(-U^{\prime}\right)=$ index $U$-index $U^{\prime}$, i.e., $\gamma(U)=\frac{1}{8}$ index $U=\frac{1}{8}$ index $U^{\prime}=\gamma\left(U^{\prime}\right)$.

Case (b) $l$ odd: Let

$$
\cdots \longrightarrow H^{l} X \stackrel{i^{*}}{\longrightarrow} H^{l} \partial X \stackrel{\delta}{\longrightarrow} H^{l+1}(X, \partial X) \longrightarrow \cdots
$$

be the exact sequence of the pair $(X, \partial X)$. We know that $H^{l} \partial X=\operatorname{Im} \tilde{r}^{*} \oplus A$. If we could choose a symplectic basis $x_{1}, \ldots, x_{s}, y_{1}, \ldots, y_{s}$ for $A \approx H_{l} U \oplus H_{l}\left(-U^{\prime}\right)$ with $x_{i} \cup x_{j}=0=y_{i} \cup y_{j}, x_{i} \cup y_{j}=\delta_{i j}$ and $\delta\left(x_{i}\right)=0$, then the argument in [11, p. 28] shows that $\gamma\left(U \underline{\underline{ \pm}}-U^{\prime}\right)=0$; hence $\gamma(U)=\gamma\left(U^{\prime}\right)$. (Note that by Poincaré-Lefschetz duality $\delta\left(x_{i}\right)=0$ implies that a sphere representing $x_{i} \cap \mu_{\partial X}$ in $\partial X$ is nullhomotopic in $X$ since $X$ is $(l-1)$-connected.) We can choose such a symplectic basis because $A$ is torsion-free (see [10, p. 27]) and $(u \cup \delta x) \cap \mu_{(X, \partial X)}=\left(i^{*} u \cup x\right) \cap \mu_{\partial X}$, for $u \in H^{l} X, x \in H^{l} \partial X$ (compare [8, p. 261]).

Now set $U=D^{n+k}$ if $n+k$ is odd.

LEMMA 5.4. $r$ may be assumed to be a homotopy equivalence if and only if $\gamma(U)=0$.

Proof. See [10, pp. 28-37] and [11, \$4.10-4.11]. It is easy to translate the arguments found there to fit our situation.

6. The maps $\omega_{i}, \phi_{i}, \partial_{i}$. In this section we define the maps $\omega_{i}, \phi_{i}, \partial_{i}$ of $\S 3$. Compare with [11, pp. 34-35]. We also prove the commutativity of diagram $(5)_{q}$. Let $n \geqq 0$.

Definition of $\omega_{1}$. Let $[f] \in \pi_{n}^{V} S O_{q}$. Define $\omega_{1}([f]) \equiv[\varepsilon, \mathscr{F}]$, where $\mathscr{F}(x, t, y)$ $=(x, t, f(x, t)(y))$.

Definition OF $\phi_{1}$. Let $[f, \mathscr{F}] \in \theta_{F}^{m, n}(V)$. Define $\phi_{1}([f, \mathscr{F}]) \equiv[f]$.

Definition of $\omega_{2}$. Let $[f, \mathscr{F}] \in \theta_{F}^{m, n}(V)$. Define $\omega_{2}([f, \mathscr{F}]) \equiv[\sigma(\mathscr{F})] \in \pi_{n}^{V} G_{q}$.

LEMMA 6.1. (a) $\omega_{1}$ and $\phi_{1}$ are well defined homomorphisms for $n \geqq 0$. (b) $\omega_{2}$ is $a$ well defined homomorphism for $n \geqq 1$. 
Proof. Easy. The homomorphism property of $\omega_{2}$ follows from the addendum to Lemma 4.3.

From now on in this section assume that $q \geqq 3, n+k \geqq 6$, and $n \geqq 1$.

Definition of $\omega_{3}$. Let $[f] \in \theta^{m, n}(V)$. Pick $\mathscr{F}^{0}$ as in Lemma 4.1(a) and define $\omega_{3}([f]) \equiv\left[\sigma\left(\mathscr{F}^{0}\right)\right] \in \pi_{n}^{V}\left(G_{q}, S O_{q}\right)$.

DEFINITION OF $\partial_{1} . \partial_{1} \equiv \partial_{4} \omega_{3}$.

LEMMA 6.2. $\omega_{3}$ and $\partial_{1}$ are well defined homomorphisms.

Proof. Apply Lemma 4.1(b) and 4.3. Observe that we can give the following description of $\partial_{1}$ : Let $[f] \in \theta^{m, n}(V)$. Choose $\mathscr{F}^{0}$ and $\alpha$ as in Lemma 4.1(a). Then $\partial_{1}([f])=[\alpha]$.

Definition of $\phi_{3}$. Let $[g] \in \pi_{n}^{V}\left(G_{q}, S O_{q}\right)$. Define $\phi_{3}([g])=\gamma\left(U^{k+n}\right)$, where $U$ is the $\pi$-manifold obtained as in $\$ 5$.

DEFINITION OF $\phi_{2}$. $\phi_{2} \equiv \phi_{3} \phi_{4}$.

LEMMA 6.3. $\phi_{2}$ and $\phi_{3}$ are well defined homomorphisms.

Proof. Lemma 5.3 shows that $\phi_{3}$ is well defined. To show that it is a homomorphism, let $[f],\left[f^{\prime}\right] \in \pi_{n}^{V}\left(G_{q}, S O_{q}\right)$. Recall that $[f] *\left[f^{\prime}\right]=[h]$, where $h: V \times I^{n}$ $\rightarrow G_{q}$, and

$$
\begin{aligned}
h\left(x,\left(t_{1}, \ldots, t_{n}\right)\right) & =f\left(x,\left(2 t_{1}, t_{2}, \ldots, t_{n}\right)\right) f^{\prime}\left(x,\left(0, t_{2}, \ldots, t_{n}\right)\right), & & 0 \leqq t_{1} \leqq \frac{1}{2}, \\
& =f^{\prime}\left(x,\left(2 t_{1}-1, t_{2}, \ldots, t_{n}\right)\right), & & \frac{1}{2} \leqq t_{1} \leqq 1 .
\end{aligned}
$$

If $W_{1} \equiv \rho(f)^{-1}(e), W_{2}=\rho\left(f^{\prime}\right)^{-1}(e)$, and $W_{3} \equiv \rho(h)^{-1}(e)$, then we may suppose as usual that the $W_{i}$ are $(k+n)$-manifolds. Furthermore, $W_{3} \approx W_{1} \cup_{g} W_{2}$, where, for $n=1$,

$$
\begin{gathered}
g: W_{1} \cap\left(V \times 1 \times S^{q-1}\right) \rightarrow W_{2} \cap\left(V \times 0 \times S^{q-1}\right), \\
g(x, 1, y)=\left(x, 0, f^{\prime}(x, 0)(y)\right),
\end{gathered}
$$

and for $n \geqq 2$,

$$
\begin{gathered}
g: W_{1} \cap\left[V \times\left(1 \times I^{n-1}\right) \times S^{q-1}\right] \rightarrow W_{2} \cap\left[V \times\left(0 \times I^{n-1}\right) \times S^{q-1}\right], \\
g\left(x,\left(1, t_{2}, \ldots, t_{n}\right), y\right)=\left(x,\left(0, t_{2}, \ldots, t_{n}\right), y\right) .
\end{gathered}
$$

Let $U^{k+n}, U^{k+n}$ be chosen for $f, f^{\prime}$, respectively, as in $\S 5$. It should be clear from the above construction $W_{3} \approx V \times I^{n} \stackrel{ \pm}{=}\left(U \stackrel{ \pm}{ \pm} U^{\prime}\right)$. Therefore $\phi_{3}\left([f] *\left[f^{\prime}\right]\right)=\phi_{3}([h])$ $=\gamma\left(U \pm U^{\prime}\right)=\gamma(U)+\gamma\left(U^{\prime}\right)=\phi_{3}([f]) * \phi_{3}\left(\left[f^{\prime}\right]\right)$. This finishes the proof.

Before going on to the next definition we need another concept. Suppose $\mathscr{F}: M^{n} \times D^{q-1} \rightarrow W^{n+q}$ is an imbedding. Then there is an essentially unique orientation preserving imbedding $\mathscr{F}^{\prime}: M^{n} \times D^{q} \rightarrow W^{n+q}$ with $\mathscr{F}^{\prime}(x, t)=\mathscr{F}(x, t)$, for $t \in D^{q-1}$. $\mathscr{F}^{\prime}$ is called the rear extension of $\mathscr{F}$ (compare [11, §1.2]).

If $(M, \mathscr{F}) \subseteq f$, then $\left(M, \frac{1}{2} \mathscr{F}\right)$ shall denote the framed imbedding $(M, \mathscr{G})$, where $\mathscr{G}(x, y)=\mathscr{F}\left(x, \frac{1}{2} y\right)$. 
Definition OF $\partial_{2}$. Let $\gamma \in P_{k+n}$. By [11, Lemma 4.6] there is a framed submanifold $\left(U^{k+n}, \mathscr{G}\right) \subseteq D^{k+n+q-1} \subseteq \operatorname{int}\left(V \times I^{n-1} \times D^{q}\right)$ such that $\gamma(U)=\gamma$ and $\sum^{k+n-1}=\partial U$ is a homotopy sphere. Assume that $D^{k+n+q-1} \cap\left(V \times I^{n-1} \times \frac{1}{2} D^{q}\right)=\varnothing$. Let

$$
\left(N^{k+n-1}, \mathscr{F}_{1}\right) \equiv\left(V \times I^{n-1} \times 0, \frac{1}{2} \mathscr{E}\right) \#\left(\Sigma, \mathscr{G}_{1}\right),
$$

where $\mathscr{G}_{1}$ is the rear extension of $\mathscr{G}$ and \# denotes (framed) connected sum (see $[3, \S 1.2])$. Clearly, the inclusion $V \times J^{n-2} \subseteq N$ is a homotopy equivalence and hence from [14, Theorem 4.1] and Lemma 4.5 there is an admissible map $f: V \times I^{n-1}$ $\rightarrow V \times I^{n-1} \times D^{q}$ with $f\left(V \times I^{n-1}\right)=N$. Define $\mathscr{F}: V \times I^{n-1} \times D^{q} \rightarrow V \times I^{n-1} \times D^{q}$ by $\mathscr{F}(x, t, y)=\mathscr{F}_{1}(f(x, t), y)$. Again using Smale theory ([14, Theorem 4.1]), we may suppose that $\mathscr{F}$ is a diffeomorphism onto $V \times I^{n-1} \times D^{q}$, so that $(f, \mathscr{F})$ is $F$-admissible. Define $\partial_{2}(\gamma)=[f, \mathscr{F}]$.

Definition of $\partial_{3}$. Define $\partial_{3} \equiv \phi_{1} \partial_{2}$.

LEMMA 6.4. $\partial_{2}$ and $\partial_{3}$ are well defined homomorphisms.

Proof. Identify $V \times I^{n-1} \times D^{q}$ with $V \times I^{n-1} \times D^{q} \times 0$ and push int $(U)$ into int $\left(V \times I^{n-1} \times D^{q} \times I\right)$ with $U \cap\left(V \times I^{n-1} \times \frac{1}{2} D^{q} \times I\right)=\varnothing$. Assume that if $\mathscr{G}^{\prime}$ is the new framing on $U$, then $\mathscr{G}^{\prime} \mid \Sigma=\mathscr{G}_{1}$. Let

$$
\left(W^{k+n}, \mathscr{H}\right) \equiv\left(V \times I^{n-1} \times 0 \times I, \frac{1}{2} \mathscr{E}\right) \pm\left(U, \mathscr{G}^{\prime}\right) .
$$

Using Lemma 5.4 and then a construction similar to the one which gave us $(f, \mathscr{F})$, it is easy to see that if $\gamma(U)=0$ then there is an $F$-connection between $(f, \mathscr{F})$ and $(\varepsilon, \mathscr{E})$ (by doing framed surgery on $W$ to make it diffeomorphic to $\left.V \times I^{n}\right)$. Thus $\partial_{2}(0)$ is well defined and equal to 0 . Furthermore, $\gamma$ is additive with respect to the connected sum operation by [11, Lemma 4.5]. Combining these facts with the definition of $\partial_{2}$, we get that $\partial_{2}$ is well defined on all of $P_{k+n}$ and a homomorphism.

Lemma 6.5. Diagram (5) $)_{q}$ of $\S 3$ is commutative up to sign.

Proof. We shall show that $\omega_{1} \partial_{4}=(-1)^{n} \partial_{2} \phi_{3}$. That the rest of the diagram commutes is immediate from the definitions.

Let $[g] \in \pi_{n+1}^{V}\left(G_{q}, S O_{q}\right), M^{k+n+1} \equiv \rho(g)^{-1}(e)$, and assume that $M$ is a manifold with framing $\mathscr{F}$ obtained from the Thom construction, where $\mathscr{F}$ is induced from the standard framing $\mathscr{E}_{1}$ of $e$ in $S^{q-1}$. Let $N^{k+n} \equiv M \cap\left(V \times I^{n} \times S^{q-1}\right)$, and let $\mathscr{F}_{1}$ be the rear extension of $\mathscr{F} \mid N$. Now push $N$ radially into $V \times I^{n} \times 0 \subseteq V \times I^{n} \times D^{q}$ along with its framing to get $\left(V \times I^{n} \times 0, \mathscr{F}_{2}\right)$. If we define $\mathscr{F}_{3}: V \times I^{n} \times D^{q} \rightarrow$ $V \times I^{n} \times D^{q}$ by $\mathscr{F}_{3}(x, t, y)=\mathscr{F}_{2}((x, t, 0), y)$, then we may assume that $\left(\varepsilon, \mathscr{F}_{3}\right)$ is an $F$-admissible pair with $\omega_{1} \partial_{4}([g])=-\left[\varepsilon, \mathscr{F}_{3}\right]$, i.e. $\mathscr{F}_{3}(x, t, y)=\left(x, t, g_{1}(x, t)^{-1}(y)\right)$, where $g_{1} \equiv g \mid V \times I^{n}$.

Now we may suppose that $M \approx\left(V \times I_{1}^{n} \times e \times I\right) \pm U^{k+n+1}$, where $U$ is a $\pi$ manifold with boundary a homotopy sphere (see Lemmas 5.1, 5.2, and 5.4). Let

$$
\left(W^{k+n+1}, \mathscr{H}\right) \equiv\left(V \times I^{n} \times 0 \times I, \frac{1}{2} \mathscr{E}\right) \pm\left((-1)^{n+1} U, \mathscr{G}\right)
$$

as in Lemma 6.4 and modify $\mathscr{H}$ so that $\mathscr{H}\left[\left(W \cap\left(V \times I^{n} \times D^{q} \times 0\right)\right) \times D^{q}\right]=V \times I^{n}$ 
$\times D^{q} \times 0$. Then $\partial_{2} \phi_{3}\left((-1)^{n+1}[g]\right)=\partial_{2}\left(\gamma\left((-1)^{n+1} U\right)\right)=\left[f, \mathscr{F}_{4}\right]$, where $f\left(V \times I^{n}\right)$ $=W \cap\left(V \times I^{n} \times D^{q} \times 0\right)$ and $\mathscr{F}_{4}(x, t, y)=\mathscr{H}(f(x, t), y)$. Finally, push $(-1)^{n+1} M$ radially into int $\left(V \times I^{n+1} \times D^{q}\right)$ with $(-1)^{n+1} \partial M$ going into $V \times \partial I^{n+1} \times 0$ and identify $V \times I^{n+1} \times D^{q}$ with $V \times I^{n} \times D^{q} \times I$. Call the resulting framed manifold $\left(M^{\prime}, \mathscr{H}_{1}\right)$. The orientation of $M^{\prime} \cap\left(V \times I^{n} \times 0 \times 1\right)$ will now agree with the orientation of $V \times I^{n} \times 0 \times 1$, because $\partial\left(I^{n+1} \times D^{q}\right)=\left(\partial I^{n+1} \times D^{q}\right) \cup(-1)^{n+1}\left(I^{n+1} \times \partial D^{q}\right)$. Note that we may assume that

$$
\mathscr{H}_{1} \mid V \times I^{n} \times 0 \times 0=\mathscr{F}_{3} \text { and } \mathscr{H}_{1} \mid\left(\left(V \times \partial I^{n} \times D^{q} \times I\right) \cup\left(V \times I^{n} \times D^{q} \times 1\right)\right)
$$

is the standard framing. Let $\left(X^{k+n+1}, \mathscr{H}_{2}\right) \equiv\left(M^{\prime}, \mathscr{H}_{1}\right) \cup_{V \times I^{n} \times 0 \times 1}(W, \mathscr{H})$. Then $X \approx V \times I^{n+1} \pm\left[(-1)^{n+1} U \pm-(-1)^{n+1} U\right]$. But $\gamma\left[(-1)^{n+1} U \pm-(-1)^{n+1} U\right]=0$. Hence by doing surgery on $X$ we may assume that $X \approx V \times I^{n+1}$. From this it is easy to construct an $F$-connection between $\left(\varepsilon, \mathscr{F}_{3}\right)$ and $\left(f, \mathscr{F}_{4}\right)$ (see Lemma 4.5 ). Thus $(-1) \omega_{1} \partial_{4}([g])=\left[\varepsilon, \mathscr{F}_{3}\right]=\left[f, \mathscr{F}_{4}\right]=(-1)^{n+1} \partial_{2} \phi_{3}([g])$.

7. Exactness. In the previous section we defined all the maps and now we show that the sequences in $\S 3$ are exact. For more details see [11], since the basic ideas behind the proofs are the same.

LEMMA 7.1. Im $\omega_{1}=\operatorname{ker} \phi_{1}$ for $n \geqq 0$.

Proof. (a) $\phi_{1} \omega_{1}=0$ : This follows immediately from the definitions. (b) ker $\phi_{1}$ $\subseteq \operatorname{Im} \omega_{1}$ : Let $[f, \mathscr{F}] \in \theta_{F}^{m, n}(V)$ and suppose that $\phi_{1}([f, \mathscr{F}])=[f]=0$. Lemma 4.2 implies that $[f, \mathscr{F}]=[\varepsilon, \mathscr{G}]$ for some $\mathscr{G}$. But then by the tubular neighborhood theorem we may assume that $\sigma(\mathscr{G}): V \times I^{n} \rightarrow S O_{q}, \sigma(\mathscr{G})\left(V \times \partial I^{n}\right)=$ identity. Clearly $\omega_{1}([\sigma(\mathscr{G})])=[f, \mathscr{F}]$.

LEMMA 7.2. $\operatorname{Im} \phi_{1}=\operatorname{ker} \partial_{1}$ for $n \geqq 1$.

Proof. (a) $\partial_{1} \phi_{1}=0$ : By Lemma 6.5, $\partial_{1} \phi_{1}=\partial_{4} \phi_{4} \omega_{2}=0 \cdot \omega_{2}=0$. (b) $\operatorname{ker} \partial_{1} \subseteq \operatorname{Im} \phi_{1}$ : We use the notation of Lemma 6.2. $\partial_{1}([f])=[\alpha]=0$ implies that there is a homotopy $h_{t}: V \times I^{n-1} \rightarrow S O_{q}$ with $h_{0}=\alpha, h_{1}\left(V \times I^{n-1}\right)=$ identity $=h_{t}\left(V \times \partial I^{n-1}\right)$. Using $h_{t}$ it should be clear how one then deforms $\mathscr{F}^{0}$ on $V \times I^{n-1}$ to get $\mathscr{F}$ with $(f, \mathscr{F})$ $F$-admissible and $\phi_{1}([\dot{f}, \mathscr{F}])=[f]$.

LeMma 7.3. Im $\partial_{1}=\operatorname{ker} \omega_{1}$ for $n \geqq 1$.

Proof. (a) $\omega_{1} \partial_{1}=0$ : Let $[f] \in \theta^{m, n}(V)$ and pick $\mathscr{F}^{0}, \alpha$ as in Lemma 4.1(a). Then $\omega_{1} \partial_{1}([f])=[\varepsilon, \mathscr{G}]$, where $\mathscr{G}(x, t, y)=(x, t, \alpha(x, t)(y))$. But it is easy to show that $[\varepsilon, \mathscr{G}]=\left[f\left|V \times I^{n-1}, \mathscr{F} 0\right| V \times I^{n-1} \times D^{q}\right]$, since $f\left(V \times I^{n-1}\right)=V \times I^{n-1} \times 0$. Now $\left(R f, R_{F}^{0} R^{-1}\right)$ is an $F$-connection between $\left(f\left|V \times I^{n-1}, \mathscr{F}^{0}\right| V \times I^{n-1} \times D^{q}\right)$ and $(\varepsilon, \mathscr{E})$; therefore, $[\varepsilon, \mathscr{G}]=0$.

(b) $\operatorname{ker} \omega_{1} \subseteq \operatorname{Im} \partial_{1}$ : Let $[g] \in \pi_{n-1}^{V} S O_{q}$. Suppose $\omega_{1}([g])=[\varepsilon, \mathscr{G}]=0$, where $\mathscr{G}(x, t, y)=(x, t, g(x, t)(y))$. Let $(H, \mathscr{H}) F$-connect $(\varepsilon, \mathscr{G})$ with $(\varepsilon, \mathscr{E})$. Define $f: V \times I^{n} \rightarrow V \times I^{n} \times D^{q}$ by $f\left(x,\left(t_{1}, \ldots, t_{n}\right)\right)=R^{-1} H\left(x,\left(t_{1}, \ldots, t_{n-1}\right), t_{n}\right) . f$ is admissible and $\partial_{1}([f])=[g]$. 
For the remainder of this section assume that $n \geqq 1, q \geqq 3$, and $n+k \geqq 6$.

LEMMA 7.4. $\operatorname{Im} \partial_{2}=\operatorname{ker} \omega_{2}$.

Proof. (a) $\omega_{2} \partial_{2}=0$ : Let $\gamma=\gamma\left(U^{k+n+1}\right) \in P_{k+n+1}$. Let $p: D_{+}^{q-1} \rightarrow D^{q-1}$ be the obvious projection in $R^{q}$ and let $\mathscr{E}^{\prime}: V \times I^{n} \times[0,2] e \times D^{q-1} \rightarrow V \times I^{n} \times 2 D^{q}$ be any framing of $V \times I^{n-1} \times[0,2] e$ such that $\mathscr{E}^{\prime}(x, t, 2 e, u)=\left(x, t, 2 e, 2 p^{-1}(u)\right)$ and the rear extension of $\mathscr{E}^{\prime} \mid V \times I^{n} \times 0$ is just $\frac{1}{2} \mathscr{E}$. Suppose that $(U, \mathscr{G}) \subseteq V \times I^{n} \times D^{q}$ with $\operatorname{Im} \mathscr{G} \cap \operatorname{Im} \mathscr{E}^{\prime}=\varnothing$. Let $\left(M^{k+n+1}, \mathscr{G}_{1}\right) \equiv(U, \mathscr{G}) \pm\left(V \times I^{n} \times[0,2] e, \mathscr{E}^{\prime}\right)$, where the (framed) connected sum is taken on int $\left(V \times I^{n} \times 0\right)$; let

$$
\left(N^{k+n}, \mathscr{G}_{2}\right) \equiv\left(\mathrm{Cl}\left(\partial M \cap \operatorname{int}\left(V \times I^{n} \times 2 D^{q}\right)\right), \mathscr{G}^{\prime}\right),
$$

with $\mathscr{G}^{\prime}$ the rear extension of $\mathscr{G}_{1} \mid \mathrm{Cl}\left(\partial M \cap\right.$ int $\left.\left(V \times I^{n} \times 2 D^{q}\right)\right)$. If $T$ is a tubular neighborhood of $N$, we may assume that $T=\mathscr{G}_{2}\left(N \times D^{q}\right)=V \times I^{n} \times D^{q}$ and $M \cap T$ $=\mathscr{G}_{2}(N \times[0,1] e)$. Let $\left(W^{k+n}, \mathscr{F}^{\prime}\right) \equiv\left(\mathscr{G}_{2}(N \times e), \mathscr{G}_{1} \mid \mathscr{G}_{2}(N \times e)\right)$ and $\left(M_{0}^{k+n+1}, \mathscr{G}_{0}\right)$ $\equiv\left(\mathrm{Cl}(M-T), \mathscr{G}_{1} \mid \mathrm{Cl}(M-T)\right)$. Now $\left((-1)^{n+1} M_{0}, \mathscr{G}_{0}\right)$ can be considered as a framed submanifold of $V \times I^{n} \times S^{q-1} \times I$ extending $\left((-1)^{n+1} W, \mathscr{F}^{\prime}\right)$. Thus $\left[t\left((-1)^{n+1} W, \mathscr{F}^{\prime}\right)\right]=0$. It follows from the definitions that if we let $f$ be an admissible map with $f\left(V \times I^{n}\right)=N$ (see Lemma 4.5) and define $\mathscr{F}: V \times I^{n} \times D^{q}$ $\rightarrow V \times I^{n} \times D^{q}$ by $\mathscr{F}(x, t, y)=\mathscr{G}_{2}(f(x, t), y)$, then $\omega_{2} \partial_{2}(\gamma)=\omega_{2}([f, \mathscr{F}])=[\sigma(\mathscr{F})]$. Let $\left((-1)^{n+1} N, \mathscr{F}_{1}\right)$, with $\operatorname{Im} \mathscr{F}_{1}=V \times I^{n} \times D^{q}$, be obtained by taking the rear extension of $\mathscr{F}^{\prime}$ and pushing it to $N$. Then analogously to Lemma 4.4,

$$
(-1)^{n+1}\left[\sigma\left(\mathscr{F}_{1}\right) f\right]=-\left[t\left((-1)^{n+1} W, \mathscr{F}^{\prime}\right)\right]=0 .
$$

But $(-1)^{n+1}\left[\sigma\left(\mathscr{F}_{1}\right) f\right]=[\sigma(\mathscr{F})]$. Compare [11, Lemma 3.8].

(b) $\operatorname{ker} \omega_{2} \subseteq \operatorname{Im} \partial_{2}$ : Suppose that $\omega_{2}([f, \mathscr{F}])=[\sigma(\mathscr{F})]=0$. Let $H: V \times I^{n} \times I \rightarrow G_{q}$ be a homotopy between $\sigma(\mathscr{F})$ and 0 , and define $W^{k+n+1} \equiv \rho(H)^{-1}(e)$. We may assume that $W$ is a framed submanifold and that $\partial W=M^{k+n} \cup V \times \partial I^{n} \times I \times e$ $\cup V \times I^{n} \times 1 \times e$ with $M \approx V \times I^{n}$. Let $r \equiv P \mid W: W \rightarrow V \times I^{n} \times 1 \times e$. Lemma 5.1 implies that we may assume $r$ to be $([(k+n+1) / 2]-1)$-connected. If $k+n+1$ is odd, then by Lemma 5.4 we may take $W \approx V \times I^{n+1}$; but then $(-1)^{n+1} W$ easily gives an $F$-connection between $(f, \mathscr{F})$ and $(\varepsilon, \mathscr{E})$ (see Lemma 4.5$)$. Hence $[f, \mathscr{F}]=0$ in this case. If $k+n+1$ is even, we can find, using Lemma 5.2, a $\pi$-manifold $U^{k+n+1}$ with boundary a homotopy sphere and $W \approx V \times I^{n+1} \pm U$. Then $\partial_{2}\left[\gamma\left((-1)^{n+1} U\right)\right]$ $=[f, \mathscr{F}]$.

LeMma 7.5. $\operatorname{Im} \omega_{2}=\operatorname{ker} \phi_{2}$.

Proof. (a) $\phi_{2} \omega_{2}=0: \quad$ Let $[f, \mathscr{F}] \in \theta_{F}^{m, n}(V) . \quad \phi_{2} \omega_{2}([f, \mathscr{F}])=\phi_{2}([\sigma(\mathscr{F})]) . \quad$ But $\sigma(\mathscr{F})^{-1}(e) \approx V \times I^{n} ;$ hence $\phi_{2}([\sigma(\mathscr{F})])=\gamma\left(D^{k+n}\right)=0$.

(b) $\operatorname{ker} \phi_{2} \subseteq \operatorname{Im} \omega_{2}$ : Let $[g] \in \pi_{n}^{V} G_{q}$. If $\phi_{2}([g])=0$, we may assume that there is an imbedding $\mathscr{H}: V \times I^{n} \times D_{+}^{q-1} \rightarrow V \times I^{n} \times S^{q-1}$ so that $\mathscr{H}(x, t, y)=(x, t, y), t \in J^{n-1}$, $\mathscr{H}(x, t, y)=\left(p_{1} \mathscr{H}(x, t, y), p_{2} \mathscr{H}(x, t, y), y\right), t \in I^{n-1}, \mathscr{H}\left(V \times I^{n} \times e\right)=W=\rho(g)^{-1}(e)$, and $[t(W, \mathscr{H})]=[g]$. If we now push $\partial W$ radially into $V \times \partial I^{n} \times 0$ and int $(W)$ into 
int $\left(V \times I^{n} \times D^{q}\right)$, it is easy to get an $F$-admissible pair $(f, \mathscr{F})$ with $[\sigma(\mathscr{F})]=$ $-[t(W, \mathscr{H})]$, using Lemma 4.4. Thus $\omega_{2}(-[f, \mathscr{F}])=[g]$.

LEMMA 7.6. $\operatorname{Im} \phi_{2}=\operatorname{ker} \partial_{2}$.

Proof. (a) $\partial_{2} \phi_{2}=0$ : By Lemma 6.5,

$$
\partial_{2} \phi_{2}=\partial_{2} \phi_{3} \phi_{4}=(-1)^{n-1} \omega_{1} \partial_{4} \phi_{4}=(-1)^{n-1} \omega_{1} \cdot 0=0 .
$$

(b) ker $\partial_{2} \subseteq \operatorname{Im} \phi_{2}$ : Let $\gamma=\gamma\left(U^{k+n}\right) \in P_{k+n}$. Let $\mathscr{E}^{\prime}$ be a framing of $V \times I^{n-1}$ $\times[0,2] e$ in $V \times I^{n-1} \times 2 D^{q}$ as in Lemma 7.4a. Let $(U, \mathscr{G}) \subseteq$ int $\left(V \times I^{n-1} \times D^{q}\right)$ with Im $\mathscr{G} \cap \operatorname{Im} \mathscr{E}^{\prime}=\varnothing$, and define $\left(W^{k+n}, \mathscr{H}\right)=(U, \mathscr{G}) \stackrel{ \pm}{=}\left(V \times I^{n-1} \times[0,2] e, \mathscr{E}^{\prime}\right)$, where the connected sum is taken on int $\left(V \times I^{n-1} \times 0\right)$; let

$$
\begin{aligned}
\left(N^{k+n-1}, \mathscr{F}_{1}\right)= & \left(\mathrm{Cl}\left(\partial W \cap \operatorname{int}\left(V \times I^{n-1} \times 2 D^{q}\right)\right),\right. \\
& \text { rear extension of } \left.\mathscr{H} \mid \mathrm{Cl}\left(\partial W \cap \operatorname{int}\left(V \times I^{n-1} \times 2 D^{q}\right)\right)\right) .
\end{aligned}
$$

We have that $\partial_{2}(\gamma)=[f, \mathscr{F}]$, where $(f, \mathscr{F})$ is obtained as in the definition of $\partial_{2}$ from $\left(N, \mathscr{F}_{1}\right)$. In fact, we may assume that $W \cap\left(V \times I^{n-1} \times D^{q}\right)=\mathscr{F}\left(V \times I^{n-1} \times[0,1] e\right)$. Let

$\left(W_{1}^{k+n}, \mathscr{H}_{1}\right) \equiv\left(W \cap\left(V \times I^{n-1} \times\left(2 D^{q}-\dot{D}^{q}\right)\right), \mathscr{H} \mid\left(W \cap\left(V \times I^{n-1} \times\left(2 D^{q}-\dot{D}^{q}\right)\right)\right)\right)$.

Now $\partial_{2}(\gamma)=0$ implies that there is an $F$-connection $(K, \mathscr{K})$ such that $(\varepsilon, \mathscr{E}) \sim_{(K, \mathscr{K})}$ $(f, \mathscr{F})$. Let $M^{k+n} \equiv \mathscr{K}\left(V \times I^{n-1} \times e \times I\right)$ and define $\mathscr{K}_{1}: M \times D_{+}^{q-1} \rightarrow V \times I^{n-1}$ $\times S^{q-1} \times I$ by $\mathscr{K}_{1}(\mathscr{K}(x, t, e, s), y)=\mathscr{K}(x, t, y, s)$. Identifying $V \times I^{n-1} \times\left(2 D^{q}-\dot{D}^{q}\right)$ with $V \times I^{n-1} \times S^{q-1} \times I$, we obtain

$$
\begin{aligned}
\left(W_{2}^{k+n}, \mathscr{H}_{2}\right) & =\left((-1)^{n} M, \mathscr{K}_{1}\right) \cup\left((-1)^{n} W_{1}, \mathscr{H}_{1}\right) \subseteq V \times I^{n-1} \times S^{q-1} \times 2 I \\
& =V \times I^{n} \times S^{q-1}
\end{aligned}
$$

and $\phi_{2}\left(\left[t\left(W_{2}, \mathscr{H}_{2}\right)\right]\right)=(-1)^{n} \gamma$. Consequently, also $\gamma$ belongs to $\operatorname{Im} \phi_{2}$.

LEMMA 7.7. $\phi_{3} \omega_{3}=0$.

Proof. Similar to Lemma 7.5(a).

THEOREM 1. Let $n \geqq 1, n+k \geqq 6, q \geqq 3$. Let $V^{k}$ be a closed, 1-connected manifold. Then the sequences $(1)_{q}-(3)_{q}$ are exact and diagram (5) commutes up to sign.

Proof. Use Lemmas 6.1-6.5, 7.1-7.7 and [11, Lemma 5.3].

Remark. To see how Proposition 5 and Theorem 5.4 of [11] follow from Theorem 1, recall our observation that cobordism classes of pairs $\left(S^{m}, K^{n}\right), K^{n}$ a homotopy sphere, are in one-to-one correspondence with cobordism classes of pairs $\left(D^{m}, D^{n}\right)$, where $\partial D^{n}$ is the usual $S^{n-1} \subset D^{m}$. Thus, letting $V^{k}=\{x\}$ in Theorem 1 we obtain the desired sequences. In fact, only $q \geqq 3, n \geqq 5$ is required (compare [13, pp. 109-113]).

8. On suspension. Now that we have the exact sequences $(1)_{q}-(4)_{q}$ we shall consider the problem as to what happens under suspension. Haefliger studies this 
question for the case of knotted spheres in [4], and we shall derive analogous results.

There are natural inclusions $S O_{q} \subseteq S O_{q+l}, G_{q} \subseteq G_{q+l}$, and $D^{q} \subseteq D^{q+l}$. These induce the obvious homomorphisms $\pi_{n}^{V} S O_{q} \rightarrow \pi_{n}^{V} S O_{q+l}, \pi_{n}^{V} G_{q} \rightarrow \pi_{n}^{V} G_{q+l}, \pi_{n}^{V}\left(G_{q}, S O_{q}\right)$ $\rightarrow \pi_{n}^{V}\left(G_{q+l}, S O_{q+l}\right), \theta^{m, n}(V) \rightarrow \theta^{m+l, n}(V)$, and $\theta_{F}^{m, n}(V) \rightarrow \theta_{F}^{m+l, n}(V)$ (by taking the rear extension of the normal frame), which we shall all denote by $s$. Along with the identity map on $P_{n+k}$ we then get, as is easily checked, homomorphisms of $(u)_{q} \rightarrow(u)_{q+l}$, for $u=1,2,3,4$ with appropriate commutativity.

For the rest of this section $q \geqq 3, n+k \geqq 6$.

LEMma 8.1. Let $[g] \in \pi_{n+1}^{V}\left(G_{q+l} ; S O_{q+l}, G_{q}\right), n \geqq 1$. Then $[g]=[f]$, where $\rho(f)^{-1}(e)$ $\approx V \times I^{n+1}$.

Proof. Assume as usual that $W^{k+n+1} \equiv \rho(g)^{-1}(e)$ is a submanifold of $V \times I^{n+1}$ $\times S^{q+l-1}$. Let $M^{k+n} \equiv \partial W \cap\left(V \times I^{n} \times S^{q+l-1}\right)$ and $g_{1} \equiv g \mid V \times I^{n}$. Then $\left[g_{1}\right]$ $\in \pi_{n}^{V}\left(G_{q}, S O_{q}\right)$ and $M=\rho\left(g_{1}\right)^{-1}(e)$. By Lemma 5.1 we may suppose that $\left(M, V \times a_{+} \times e\right)$ is $([(k+n) / 2]-1)$-connected. Consider the exact sequence

$$
\cdots \longrightarrow \pi_{n+1}^{v}\left(G_{q+l} ; S O_{q+l}, G_{q}\right) \stackrel{\partial}{\longrightarrow} \pi_{n}^{v}\left(G_{q}, S O_{q}\right) \stackrel{i_{\#}}{\longrightarrow} \pi_{n}^{v}\left(G_{q+l}, S O_{q+l}\right) \longrightarrow \cdots
$$

We have that $\phi_{3}\left(\left[g_{1}\right]\right)=\phi_{3} i_{\#}\left(\left[g_{1}\right]\right)=\phi_{3} i_{\#} \partial([g])=\phi_{3}(0)=0$, and by Lemma 5.4 we may actually assume that $M \approx V \times I^{n}$. Using Lemma 5.1 on $W$ we can make $\left(W, V \times a_{+} \times e\right)([(k+n+1) / 2]-1)$-connected, and by Lemma 5.2 and 5.4, the only obstruction to making $W \approx V \times I^{n+1}$ is an element $\gamma \in P_{k+n+1}$. Let $\gamma=\gamma\left(U^{k+n+1}\right)$, where $U$ is a $\pi$-manifold with boundary a homotopy sphere. As we noted earlier, there is a framed imbedding $(U, \mathscr{F}) \subseteq V \times I^{n+1} \times S^{q-1}$ with $U \cap\left(V \times \partial I^{n+1} \times S^{q-1}\right)$ $=\partial U$ (see [11, Lemma 4.6]). Let $\mathscr{E}_{1}$ be the standard frame of $V \times I^{n+1} \times e$ in $V \times I^{n+1} \times S^{q-1}$ and form $\left(N^{k+n+1}, \mathscr{H}\right) \equiv\left(V \times I^{n+1} \times e, \mathscr{E}_{1}\right) \pm(-U, \mathscr{F})$. But $[t(N, \mathscr{H})]=0 \in \pi_{n+1}^{V}\left(G_{q+l} ; S O_{q+l}, G_{q}\right)$. Thus, if we let $f \equiv t(n, \mathscr{H}) \cdot g$, then $[f]=[g]$ and we can make $\rho(f)^{-1}(e) \equiv V \times I^{n+1}$.

Definition of $\omega_{5}$. Let $[g] \in \pi_{n+1}^{V}\left(G_{q+l} ; S O_{q+l}, G_{q}\right)$. By Lemma 8.1, $[g]=[f]$, where $\rho(f)^{-1}(e) \approx V \times I^{n+1}$. Let

$$
M^{k+n} \equiv \rho(f)^{-1}(e) \cap V \times I^{n} \times S^{q+l-1} \subseteq V \times I^{n} \times S^{q-1} .
$$

Push $M$ radially into an $M_{0}^{k+n} \subseteq V \times I^{n} \times D^{q}$, where $\partial M_{0}=V \times \partial I^{n} \times 0$ and int $M_{0}$ $\subseteq$ int $\left(V \times I^{n} \times D^{q}\right)$. This is possible because $\left[f \mid V \times I^{n}\right] \in \pi_{n}^{V}\left(G_{q}, S O_{q}\right)$. By Lemma 4.5 there is an admissible map $h: V \times I^{n} \rightarrow V \times I^{n} \times D^{q}$ with $h\left(V \times I^{n}\right)=M_{0}$ (note that $\left.M_{0} \approx M \approx V \times I^{n}\right)$. Define $\omega_{5}([g]) \equiv[h]$.

Definition of $\phi_{5}$. Define $\phi_{5}$ to be the composition

$$
\theta^{m+l, n}(V) \underset{\omega_{3}}{\longrightarrow} \pi_{n}^{V}\left(G_{q+l}, S O_{q+l}\right) \longrightarrow \pi_{n}^{V}\left(G_{q+l} ; S O_{q+l}, G_{q}\right) .
$$

LEMMA 8.2. $\omega_{5}$ and $\phi_{5}$ are well defined homomorphisms for $n \geqq 1$.

Proof. Suppose that $\left[g^{\prime}\right]=[g] \in \pi_{n+1}^{V}\left(G_{q+l} ; S O_{q+l}, G_{q}\right)$. Define $f^{\prime}, M^{k+n^{\prime}}, M_{0}^{k+n^{\prime}}$, 
$h^{\prime}$, with respect to $g^{\prime}$, similarly to $f, M, M_{0}, h$. Let $\alpha: V \times I^{n+1} \times I \rightarrow G_{q+l}$ be a homotopy between $f$ and $f^{\prime}$ such that $\alpha(x, t, 0)=f(x, t)$ and $\alpha(x, t, 1)=f^{\prime}(x, t)$. Set

$$
X^{k+n+2} \equiv \rho(\alpha)^{-1}(e) \subseteq V \times I^{n+1} \times I \times S^{q+l-1}
$$

and

$$
\tilde{M}^{k+n+1} \equiv \rho\left(\alpha \mid V \times I^{n} \times I\right)^{-1}(e) \subseteq V \times I^{n} \times I \times S^{q-1} .
$$

Let us assume for the moment that $\tilde{M} \approx V \times I^{n+1}$. Let

$$
\begin{gathered}
\tilde{M}^{k+n+1^{\prime}} \subseteq V \times I^{n} \times I \times D^{q}, \quad \partial \tilde{M}^{\prime}=M_{0} \cup\left(V \times \partial I^{n} \times I \times 0\right) \cup M_{0}^{\prime}, \\
\text { int } \tilde{M}^{\prime} \subseteq \operatorname{int}\left(V \times I^{n} \times I \times D^{q}\right)
\end{gathered}
$$

be obtained by pushing $\tilde{M}$ radially into $V \times I^{n} \times I \times D^{q} . R\left(\tilde{M}^{\prime}\right)$ easily gives a connection between $h$ and $h^{\prime}$ (compare Lemma 4.5). Therefore $\omega_{5}([g])=[h]=\left[h^{\prime}\right]$ $=\omega_{5}\left(\left[g^{\prime}\right]\right)$, and $\omega_{5}$ is well defined.

In order to show that we may pick $\alpha$ so that $\tilde{M} \approx V \times I^{n+1}$, we first apply Lemma 5.1 to get $\left(\tilde{M}, V \times a_{+} \times 0 \times e\right)([(k+n+1) / 2]-1)$-connected. Since $\mathrm{Cl}(\partial X-\tilde{M})$ $\approx V \times I^{n+1}$, the proof of Lemma 5.3 shows that the obstructions in $P_{k+n+1}$ to making $\tilde{M} \approx V \times I^{n+1}$ vanish and Lemma 5.4 applies. (We are in essence using a relative version of [11, Lemma 4.4].) This proves that $\omega_{5}$ is well defined, but straightforward application of the definitions involved shows that $\omega_{5}$ is also a homomorphism.

Let

$$
\begin{gathered}
\cdots \longrightarrow \pi_{n+1}^{V}\left(G_{q}, S O_{q}\right) \stackrel{i}{\longrightarrow} \pi_{n+1}^{V}\left(G_{q+l}, S O_{q+l}\right) \stackrel{j}{\longrightarrow} \pi_{n+1}^{V}\left(G_{q+l} ; S O_{q+l}, G_{q}\right) \\
\stackrel{\partial}{\longrightarrow} \pi_{n}^{V}\left(G_{q}, S O_{q}\right) \longrightarrow \cdots
\end{gathered}
$$

be the exact sequence of the triad $\left(G_{q+l} ; S O_{q+l}, G_{q}\right)$.

TheOREM 2. Let $q \geqq 3, n \geqq 2, n+k \geqq 6$. Let $V^{k}$ be a closed, 1-connected manifold. Then the sequence $(6)_{q}$ is exact. Furthermore, $\partial=-\omega_{3} \omega_{5}$.

Proof. (a) $s \omega_{5}=0$ : Let $[g] \in \pi_{n+1}^{V}\left(G_{q+l} ; S O_{q+l}, G_{q}\right)$. Using the notation in the definition of $\omega_{5}$ one sees that one can also push $\rho(f)^{-1}(e)$ radially into $V \times I^{n+1}$ $\times D^{q+l}$ to obtain $W_{1}^{k+n+1} \subseteq V \times I^{n+1} \times D^{q+l}$ such that

$$
\partial W_{1}=M_{0} \cup\left(V \times \partial I^{n} \times I \times 0\right) \cup\left(V \times I^{n} \times 1 \times 0\right)
$$

and int $W_{1} \subseteq$ int $\left(V \times I^{n+1} \times D^{q+l}\right)$. Since $W_{1} \approx V \times I^{n+1}, R\left(W_{1}\right)$ gives us a connection between $h$ and $\varepsilon$ (compare Lemma 4.5). Thus, $s \omega_{5}([g])=s([h])=0$.

(b) $\operatorname{ker} s \subseteq \operatorname{Im} \omega_{5}$ : Let $[f] \in \theta^{m, n}(V)$ and suppose $s([f])=0$, i.e., there is a connection $H$ between $f$ and $\varepsilon$. Pick $\mathscr{F}^{0}: V \times I^{n} \times D^{q} \rightarrow V \times I^{n} \times D^{q}$ as in Lemma 4.1(a). Let $\tilde{F}^{0}: V \times I^{n} \times D^{q+l} \rightarrow V \times I^{n} \times D^{q+l}$ be the rear suspension of $\mathscr{F}^{0}$. Now let $\mathscr{H}^{0}: V \times I^{n} \times D^{q+l} \times I \rightarrow V \times I^{n} \times D^{q+l} \times I$ be as in Lemma 4.1(b) with respect to $H,\left(f, \tilde{\mathscr{F}}^{0}\right)$, and $(\varepsilon, \mathscr{E})$. Then $\left[\sigma\left(p_{3} \mathscr{H}^{0} R\right)\right] \in \pi_{n+1}^{v}\left(G_{q+l} ; S O_{q+l}, G_{q}\right)$, and it follows immeriately from the definitions that $\omega_{5}\left(\left[\sigma\left(p_{3} \mathscr{H}^{0} R\right)\right]\right)=[f]$. 
(c) $\phi_{5} s=0: \phi_{5} s=j \omega_{3} s=j i \omega_{3}=0 \cdot \omega_{3}=0$.

(d) $\operatorname{ker} \phi_{5} \subseteq \operatorname{Im} s$ : We must do a little diagram chasing. Let $x \in \theta^{m+l, n}(V)$ and $\phi_{5}(x)=0$. Then $j \omega_{3}(x)=0$, and there is a $y \in \pi_{n}^{V}\left(G_{q}, S O_{q}\right)$ such that $i(y)=\omega_{3}(x)$. Now $\phi_{3}(y)=\phi_{3} i(y)=\phi_{3} \omega_{3}(x)=0$; so there is a $z \in \theta^{m, n}(V)$ with $\omega_{3}(z)=y . \omega_{3}(x-s(z))$ $=\omega_{3}(x)-\omega_{3} s(z)=\omega_{3}(x)-i \omega_{3}(z)=\omega_{3}(x)-i(y)=\omega_{3}(x)-\omega_{3}(x)=0$. Hence we can find a $u \in P_{k+n+1}$ and $\partial_{3}(u)=x-s(z)$. Finally, observe that $s\left(z+\partial_{3}(u)\right)=s(z)$ $+(x-s(z))=x$.

(e) $\omega_{5} \phi_{5}=0: \omega_{5} \phi_{5}=\omega_{5} j \omega_{3}$. But it follows immediately from the definitions that $\omega_{5} j=0$. Hence $\omega_{5} \phi_{5}=0 \cdot \omega_{3}=0$.

(f) $\operatorname{ker} \omega_{5} \subseteq \operatorname{Im} \phi_{5}$ : Let $[g] \in \pi_{n}^{V}\left(G_{q+l} ; S O_{q+l}, G_{q}\right)$, and suppose $\omega_{5}([g])=[h]=0$ (using the notation in the definition of $\omega_{5}$ ). Pick $\mathscr{F}^{0}$ with respect to $h$ as in Lemma 4.1(a) and let $f_{1} \equiv f \mid V \times I^{n-1}$. Then

$$
\left[\sigma\left(\mathscr{F}^{0}\right)\right]=-\left[f_{1}\right] \in \pi_{n-1}^{V}\left(G_{q}, S O_{q}\right) .
$$

Assume this for the moment and let $H: V \times I^{n-1} \times I \rightarrow V \times I^{n-1} \times D^{q} \times I$ be a connection between $\varepsilon$ and $h$. Pick $\mathscr{H}^{0}: V \times I^{n-1} \times D^{q} \times I \rightarrow V \times I^{n-1} \times D^{q-1} \times I$ with respect to $H,(\varepsilon, \mathscr{E})$, and $\left(h, \mathscr{F}^{0}\right)$, as in Lemma 4.1(b). Then $\sigma\left(R^{-1} \mathscr{H}^{0} R\right)$ is a homotopy between $\sigma\left(\mathscr{F}^{0}\right)$ and the trivial map. Thus $\sigma\left(R^{-1} \mathscr{H}^{0-1} R\right)$ is a homotopy between $\left[f_{1}\right]$ and the trivial map in $\pi_{n-1}^{V}\left(G_{q}, S O_{q}\right)$. Now $\left(p_{3} R^{-1} \mathscr{H}^{0^{-1}} R\right)^{-1}(e)$ $\approx V \times I^{n}$ and so we could have assumed at the beginning that $f\left(V \times I^{n-1}\right)=$ identity. It follows that $[f] \in \pi_{n}^{V}\left(G_{q+l}, S O_{q+l}\right)$ and $\phi_{3}([f])=0$. Hence there is an $x \in \theta^{m+l, n}(V)$ such that $\omega_{3}(x)=[f]$, and so $\phi_{5}(x)=j \omega_{3}(x)=[f]$.

We must still prove $\left(^{*}\right)$. Now $M$ is actually a framed submanifold of $V \times I^{n-1}$ $\times S^{q-1}$ with its framing $\mathscr{F}$ induced from the standard framing of $e$ in $S^{q-1} . M_{0}$ may be given a framing $\mathscr{F}^{\prime}$, induced from the rear extension of $\mathscr{F}$, with $\mathscr{F}^{\prime}\left(M_{0} \times D^{q}\right)$ $=V \times I^{n-1} \times D^{q}$. Define $\mathscr{F}_{1}: V \times I^{n-1} \times D^{q} \rightarrow V \times I^{n-1} \times D^{q}$ by $\mathscr{F}_{1}(x, t, y)=$ $\mathscr{F}^{\prime}(h(x, t), y)$. We may assume that $\mathscr{F}_{1}$ has the following properties:

(i) $\sigma\left(\mathscr{F}_{1}^{-1}\right)(x, t)=f_{1}(x, t)$, for $t \in \partial I^{n-1}$,

(ii) $\sigma\left(\mathscr{F}_{1}^{-1}\right)(x, t)(y)=f_{1}(x, t)(y)$, for $t \in I^{n-1}, y \in D_{+}^{q-1}$.

(Property (ii) is just part of the statement that maps can be obtained via a Thom construction.) By shrinking discs $D_{x, t} \subseteq D_{-}^{q-1}$, on which $\sigma\left(\mathscr{F}_{1}^{-1}\right)(x, t)$ and $f_{1}(x, t)$ do not agree, to a point, we see that $\sigma\left(\mathscr{F}_{1}^{-1}\right) \simeq f_{1}$. Finally, note that $\sigma\left(\mathscr{F}_{1}\right) \simeq \sigma\left(\mathscr{F}^{0}\right)$ by Lemma 4.1(b). This finishes the proof that ker $\omega_{5} \subseteq \operatorname{Im} \phi_{5}$.

(g) $\partial=-\omega_{3} \omega_{5}$ : This follows from $\left(^{*}\right)$ in part (f), because in that notation, $\omega_{3} \omega_{5}([g])=\omega_{3}([h])=\left[\sigma\left(\mathscr{F}^{0}\right)\right]=-\left[f_{1}\right]=-\partial([f])=-\partial([g])$.

We now come to the sequence $(7)_{q}$, but we shall only give the necessary definitions and no proofs because they are so similar to those given for $(6)_{q}$.

Definition of $\omega_{6}$. Let $[g] \in \tilde{\pi}_{n+1}^{V}\left(G_{q+l} ; S O_{q+l}, G_{q}\right), n \geqq 1$, and, except for this change, make the same constructions as in the definition of $\omega_{5}$ (note that we may assume $\left.[f] \in \tilde{\pi}_{n+1}^{V}\left(G_{q+l} ; S O_{q+l}, G_{q}\right)\right) . M$ is actually a framed manifold and by taking the rear extension we get a framing on $M_{0}$. This in turn induces $\mathscr{H}$ so that $(h, \mathscr{H})$ is $F$-admissible (because $f\left(V \times \partial I^{n} \times I\right)=$ identity). Define $\omega_{6}([g])=[h, \mathscr{H}]$. 
Definition of $\phi_{6}$. Define $\phi_{6}$ to be the composition of

$$
\theta_{F}^{m+l, n}(V) \underset{\omega_{2}}{\longrightarrow} \pi_{n}^{V} G_{q+l} \longrightarrow \pi_{n}^{V}\left(G_{q+l}, S O_{q+l}\right) \longrightarrow \tilde{\pi}_{n}^{V}\left(G_{q+l} ; S O_{q+l}, G_{q}\right) .
$$

LeMmA 8.3. $\omega_{6}$ and $\phi_{6}$ are well defined homomorphisms for $n \geqq 1$.

Proof. Similar to that of Lemma 8.2.

NoTE. $\phi_{6}$ is also equal to the composition of

$$
\theta_{F}^{m+l, n}(V) \underset{\phi_{1}}{\longrightarrow} \theta^{m+l, n}(V) \underset{\omega_{3}}{\longrightarrow} \pi_{n}^{V}\left(G_{q+l}, S O_{q+l}\right) \longrightarrow \tilde{\pi}_{n}^{V}\left(G_{q+l} ; S O_{q+l}, G_{q}\right) .
$$

THEOREM 3. Let $q \geqq 3, n \geqq 2, n+k \geqq 6$. Let $V^{k}$ be a closed, 1-connected manifold. Then the sequence $(7)_{q}$ is exact. Furthermore, $\partial^{\prime}=-\omega_{2} \omega_{6}$, where

$$
\partial^{\prime}: \tilde{\pi}_{n+1}^{V}\left(G_{q+l} ; S O_{q+l}, G_{q}\right) \rightarrow \pi_{n}^{V} G_{q}
$$

is the boundary homomorphism.

Proof. Similar to that of Theorem 2.

LEMMA 8.4. $s: \theta^{m, n}(V) \rightarrow \theta^{m+1, n}(V)$ is an isomorphism if $k+n<2 q-3$; if $q>k+n+1$, then also $s: \theta_{F}^{m, n}(V) \rightarrow \theta_{F}^{m+1, n}(V)$ is an isomorphism.

Proof. Indeed, $\pi_{j+1}\left(G_{q+1} ; S O_{q+1}, G_{q}\right)=0$ for $j<2 q-3$ (see [6] or [4, \$7.7]), and so by standard obstruction theory $\pi_{j+1}^{V}\left(G_{q+1} ; S O_{q+1}, G_{q}\right)=0$ for $k+j<2 q-3$. Sequence $(6)_{q}$ now gives the result for $\theta^{m, n}(V)$. Since $\pi_{j} G_{q} \rightarrow \pi, G_{q+1}$ is an isomorphism if $j+1<q$, we get the second part of the lemma by using the Five Lemma and the suspension $(2)_{q} \rightarrow(2)_{q+1}$.

Lemma 8.4 enables us to define stable groups.

Definition. Let $q>k+n+1$. Define $\theta^{n}(V) \equiv \theta^{m, n}(V)$ and $\theta_{F}^{n}(V) \equiv \theta_{F}^{m, n}(V)$.

This concludes our general development of the relative knot groups $\theta^{m, n}(V)$ and $\theta_{F}^{m, n}(V)$. One can define analogues of twist-spinning operations (see W. C. Hsiang, and B. J. Sanderson, Twist-spinning spheres in spheres, Illinois J. Math. 9 (1965), 651-659). One can also extend some other results of [4], e.g., it is possible to define natural analogues of the groups $C_{n}^{q}$ and show that they are isomorphic to $\pi_{n+1}^{V}\left(G_{q+l} ; S O_{q+l}, G_{q}\right)$ for large $l$. In this way one reobtains some special cases of Haefliger's recent work on thickenings of manifolds. Finally, we would like to mention that the methods of this section apply to give alternate proofs of $[4$, Theorems 3.4 and 5.7] (which are much simpler, given $\$ 5$ and its prerequisites).

9. Tangential equivalences. We now want to show how the sequences of $\$ 3$ are related to the question of tangential homotopy equivalences between manifolds. Let $V^{k}$ be a manifold. Define $G_{V}$ to be the space of homotopy equivalences of $V$ of degree $1, D_{V}$ to be the space of diffeomorphisms in $G_{V}$, and $D_{V}^{0}$ to be the space of diffeomorphisms of $V$ which are homotopic to the identity. Note that $\pi_{0} D_{V}^{0}$ and $\pi_{1}\left(G_{V}, D_{V}\right)$ are groups. We have the following exact sequence:

$$
\cdots \rightarrow \pi_{1} D_{V} \rightarrow \pi_{1} G_{V} \rightarrow \pi_{1}\left(G_{V}, D_{V}\right) \rightarrow \pi_{0} D_{V}^{0} \rightarrow 0 .
$$


Definition of $\lambda^{\prime}$. Let $[g] \in \pi_{1}\left(G_{V}, D_{V}\right)$ and define $g_{1}: V \times I \rightarrow V \times I \times D^{N}, N$ large, by $g_{1}(x, t)=(g(t)(x), t, 0)$. By Whitney's imbedding theorem there is an imbedding $f: V \times I \rightarrow V \times I \times D^{N}$, such that

(i) $f$ is admissible,

(ii) $f(x, 1)=(x, 1,0), f(x, 0)=(g(0)(x), 0,0)$,

(iii) if we define $f_{0}: I \rightarrow G_{V}$ by $f_{0}(t)(x)=p_{1} f(x, t)$, then $\left[f_{0}\right]=[g] \in \pi_{1}\left(G_{V}, D_{V}\right)$. Define $\lambda^{\prime}: \pi_{1}\left(G_{V}, D_{V}\right) \rightarrow \theta^{1}(V)$ by $\lambda^{\prime}([g])=[f]$.

LemMa 9.1. Let $V^{k}$ be a closed, 2-connected manifold with $k \geqq 5$. Suppose $[f]=$ $\left[f^{\prime}\right] \in \theta^{1}(V)$. Then there is a connection $H: V \times I \times I \rightarrow V \times I \times D^{N} \times I$ between $f$ and $f^{\prime}$ with $H(V \times 0 \times s)=V \times 0 \times 0 \times s$.

Proof. Assume first that $f=\varepsilon$, and let $H_{1}$ be any connection between $\varepsilon$ and $f^{\prime}$. Then $H_{1}(V \times 0 \times I)=V \times 0 \times 0 \times I$. Define $H_{2}: V \times I \rightarrow V \times I$ by

$$
H_{2}(x, t)=\left(p_{1} H_{1}(x, 0, t), p_{4} H_{1}(x, 0, t)\right) .
$$

$H_{2}$ is a pseudo-isotopy, i.e., $H_{2}$ is a diffeomorphism of $V \times I$ and $H_{2}(x, 0)=(x, 0)$. We need the following theorem:

Theorem (Cerf-UnPUblished). Let $V^{k}$ be an oriented, compact, closed, and 2connected $C^{\infty}$-manifold with $k \geqq 5$. Then $\pi_{0} \mathscr{P}=0$, where $\mathscr{P}$ is the space of pseudoisotopies of $V \times I$ with the $C^{\infty}$-topology.

Using Cerf's theorem one can deform $\mathrm{H}_{2}$ to the identity, and from this it is easy to find a connection $H$ between $\varepsilon$ and $f^{\prime}$ satisfying the lemma. A similar proof works for arbitrary $f$.

THEOREM 4. Let $V^{k}$ be a closed, 2-connected manifold with $k \geqq 5$. Then $\lambda^{\prime}$ is a well defined isomorphism.

Proof. Suppose $[g]=\left[g^{\prime}\right] \in \pi_{1}\left(G_{V}, D_{V}\right)$. Choose $f, f^{\prime}$ with respect to $g, g^{\prime}$, as in the definition of $\lambda^{\prime}$. Let $h_{t}$ be a homotopy between $g$ and $g^{\prime}$ with $h_{0}=g$ and $h_{1}=g^{\prime}$. Define $H: V \times I \times I \rightarrow V \times I \times D^{N} \times I$ by $H(x, t, s)=\left(h_{s}(t)(x), t, 0, s\right)$. Applying Whitney's imbedding theorem we can get an imbedding $\tilde{H}$ approximating $H$ which is a connection between $f$ and $f^{\prime}$. Thus, $\lambda([g])=[f]=\left[f^{\prime}\right]=\lambda\left(\left[g^{\prime}\right]\right)$ and $\lambda^{\prime}$ is well defined. The homomorphism property follows directly from the definition of multiplication in $\pi_{1}\left(G_{V}, D_{V}\right)$ and $\theta^{1}(V)$.

Next, suppose that $\lambda^{\prime}([g])=0$. Again let $f, f_{0}$ be as in the definition of $\lambda^{\prime}$. Since $[f]=0$, Lemma 9.1 guarantees the existence of a connection $H$ so that $H(V \times 0 \times s)$ $=V \times 0 \times 0 \times s$. Define $h_{s}: I \rightarrow G_{V}$ by $h_{s}(t)(x)=p_{1} H(x, t, s)$. Then $h_{0}=f_{0} \simeq g, h_{1}(I)$ $=$ identity, and $h_{s}(0) \in D_{V}$, for all $s$. Therefore, $h_{s}$ is a homotopy between $g$ and the trivial map, i.e., $[g]=0$. This shows that $\lambda^{\prime}$ is an injection.

Finally, let $[f] \in \theta^{1}(V), f: V \times I \rightarrow V \times I \times D^{N}$, and define $g: I \rightarrow G_{V}$ by $g(t)(x)$ $=p_{1} f(x, t)$. Clearly $\lambda^{\prime}([g])=[f]$, showing that $\lambda^{\prime}$ is surjective and completing the proof of the theorem. 
Definition of $\lambda, \lambda_{0}$. Define $\lambda, \lambda_{0}$ to be the composition of

$$
\theta^{1}(V) \stackrel{\lambda^{\prime-1}}{\longrightarrow} \pi_{1}\left(G_{V}, D_{V}\right) \longrightarrow \pi_{0} D_{V}^{0}, \pi_{1} G_{V} \longrightarrow \pi_{1}\left(G_{V}, D_{V}\right) \stackrel{\lambda^{\prime}}{\longrightarrow} \theta^{1}(V),
$$

respectively.

Note. If $[f] \in \theta^{1}(V)$, then $\lambda([f])=\left[p_{1} f(, 0)\right]$.

Now let $G \equiv \bigcup^{q} G_{q}, S O \equiv \bigcup^{q} S O_{q}$.

CoROllary 4.1. If $V^{k}$ is a closed, 2-connected manifold with $k \geqq 5$, we have the following diagram of exact sequences

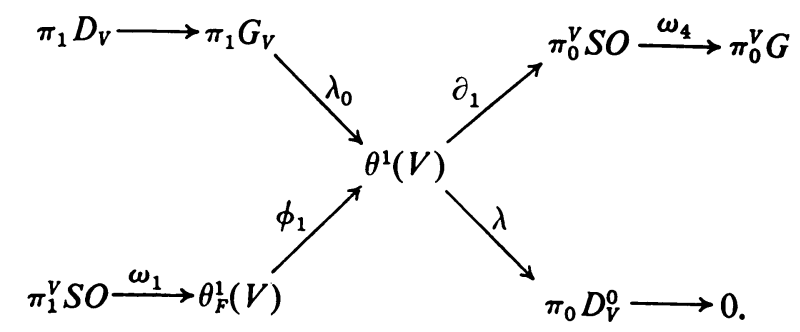

To see the connection between Corollary 4.1 and stable tangential homotopy equivalence we first make some definitions and prove a lemma before stating the main theorem.

Let $f: M^{k+1} \rightarrow W^{l}$.

DEFINITION. $f$ is a stable tangential homotopy equivalence (s.t.h.e.) if $f$ is a homotopy equivalence and $f^{*}\left(\right.$ stable $\left.\tau_{W}\right)=$ stable $\tau_{M}$. (The second condition is equivalent to saying that $f$ is normal.)

$f$ is a partial tangential homotopy equivalence (p.t.h.e.) if $f$ is a homotopy equivalence and for every map $\phi: K \rightarrow M$ of a polyhedron $K$ of dimension $\leqq m$ $=[(k+1) / 2]$ we have $\phi^{*} \tau_{M}=\phi^{*} f^{*} \tau_{W}$.

$\theta\left(M^{n}\right) \equiv$ diffeomorphism classes of $W^{m}$ for which there is a p.t.h.e. $f: W \rightarrow M$.

$\theta_{T}\left(M^{n}\right) \equiv$ diffeomorphism classes of $W^{m}$ for which there is a s.t.h.e. $f: W \rightarrow M$.

Definition. $M$ satisfies (*) if, for some handle decomposition, $M$ has no handles in dimension $m$, i.e., there is a nice Morse function $\eta: M \rightarrow \boldsymbol{R}$ (see [13, p. 44]) with $M_{m-1}(\eta) \approx M_{m}(\eta)$, where $M_{i}(\eta)=\eta^{-1}[0, i+1 / 2]$.

From now on assume that $M^{k+1}$ is closed, 1-connected, and $k \geqq 5$. Then minimal nice Morse functions $\eta$ exist by $[14$, Theorem 6.1$]$ and condition $\left({ }^{*}\right)$ is independent of any particular choice of such $\eta$. Henceforth fix $\eta$ and let $M_{i} \equiv M_{i}(\eta)$.

Lemma 9.2. Let $M^{k+1}$ satisfy $\left(^{*}\right)$, and set $U^{k+1} \equiv M_{m}$. Then

(a) $M \approx U_{F_{0}}$, for some diffeomorphism $F_{0}: \partial U \rightarrow \partial U$, where $U_{F_{0}}=U \bigcup_{F_{0}} U$.

(b) $W^{k+1} \in \theta(M)$ if and only if $W \approx U_{F}$ for some diffeomorphism $F: \partial U \rightarrow \partial U$ with $F \simeq F_{0}$.

(c) Suppose $M \approx D U \equiv$ double of $U$. Then $M \approx U_{F}$ if and only if $F$ extends to a diffeomorphism of $U \rightarrow U$. 
Proof. First observe that the diffeomorphism class of $U$ does not depend on the particular $\eta$ which is chosen, because $M$ satisfies (*) and hence $H_{m} M$ has no torsion (see [1, Theorem 1]).

(a) Comparing $\eta$ and $n-\eta$, [1, Theorem 1] gives us a diffeomorphism $F^{\prime}: M \rightarrow M$ such that $F^{\prime} \approx$ identity and $F^{\prime}(U)=\mathrm{Cl}(M-U)$. Set $F_{0} \equiv F^{\prime} \mid \partial U$. Then $M \approx U_{F_{0}}$.

(b) Let $h: M \rightarrow W^{k+1} \in \theta(M)$ be a p.t.h.e. By a proof similar to that of [14, Theorem 7.1], we may suppose that $h \mid U$ and $h \mid \mathrm{Cl}\left(M-M_{m+1}\right)$ are imbeddings. We may also suppose that $h\left(\mathrm{Cl}\left(M_{m+1}-U\right)\right) \subseteq\left(W-h\left[U \cup\left(M-M_{m+1}\right)\right]\right) \approx_{L_{0}}^{\leftarrow}$ $\partial U \times I$, with $L_{0}(x, 1)=h(x)$. Let $\partial U \times I \approx \vec{L}_{1} \mathrm{Cl}\left(M_{m+1}-U\right)$ with $L_{1}(x, 1)=x$. Define $K_{t}: \partial U \rightarrow \partial U$ by $K_{t}(x)=p_{1} L_{0}^{-1} h L_{1}\left(F_{0}(x), t\right)$, and set $F \equiv K_{0}$. Then $W \approx U_{F}$ and $K_{t}$ is a homotopy between $F$ and $F_{0}$. The converse is clear.

(c) Suppose that $M \approx_{L_{0}} D U$. Let $M \approx_{L_{1}}^{-} U_{F}$. By an application of [1, Theorem 1] we may assume that $L_{0} L_{1}(U)=U$. Thus, suppose without loss of generality that $L_{2} \mid U=$ identity, where $L_{2}: U_{F} \rightarrow D U$ is the diffeomorphism $L_{0} L_{1}$ followed by the inverse of the double of $L_{0} L_{1} \mid U: U \rightarrow U$. By considering $L_{2} \mid \mathrm{Cl}\left(U_{F}-U\right)$, it follows easily that $F$ extends to $U$. The converse is also easy.

The next theorem is what we are after.

THEOREM 5. Let $M^{k+1}$ be a closed, 2-connected manifold satisfying $\left(^{*}\right)$ with $k \geqq 5$. Set $U^{k+1} \equiv M_{m}(\eta)$, where $m=[(k+1) / 2]$, and let $V^{k} \equiv \partial U$. By Lemma 9.2(a), $M \approx U_{F_{0}}$. Let $W^{k+1} \in \theta(M)$. Then $W \in \theta_{T}(M)$ if and only if $W \approx U_{F}$, where $\left[F F_{0}^{-1}\right]$ $\in \operatorname{Im} \lambda \phi_{1} \subseteq \pi_{0} D_{V}^{0}$.

Proof. Suppose $W \in \theta_{T}(M)$ and let $h: M \rightarrow W$ be a s.t.h.e. Let $U^{k+1^{\prime}} \equiv$ $\mathrm{Cl}\left(M-M_{m+1}\right)$. Since dimension $\left(U \cup U^{\prime}\right) \leqq m$, there is a minimal nice Morse function $\eta_{1}: M \rightarrow R$ such that $A \equiv \eta_{1}^{-1}[0, m]$ contains $U \cup U^{\prime}$ in its interior. As before, we may assume that $h \mid A$ is an imbedding and also that $h(M-A) \subseteq W-h(A)$. We can extend $h \mid A$ to a diffeomorphism $H_{1}: A \times D^{N} \rightarrow h(A) \times D^{N}, N$ large, by setting $H_{1}(x, y)=(h(x), y)$. But the proof of [14, Theorem 7.1] shows that we may extend $H_{1}$ to a diffeomorphism $H: M \times D^{N} \rightarrow W \times D^{N}$. Now let $V \times I \approx_{L_{0}}$ [ $W-h\left(\dot{U} \cup \dot{U}^{\prime}\right)$ ] and $V \times I \approx_{L_{1}} \mathrm{Cl}\left(M_{m+1}-U\right)$ with $L_{0}(x, 1)=h(x)$ and $L_{1}(x, 1)=x$. Define $L_{2}: V \times I \times D^{N} \underset{\approx}{\rightarrow}\left[W-h\left(\dot{U} \cup \dot{U}^{\prime}\right)\right] \times D^{N}$ and $L_{3}: V \times I \times D^{N} \rightarrow \mathrm{Cl}\left(M_{m+1}-U\right)$ $\times D^{N}$. by $L_{2}(x, t, y) \equiv\left(L_{0}(x, t), y\right)$ and $L_{3}(x, t, y) \equiv\left(L_{1}(x, t), y\right)$. Finally, define $f: V \times I \rightarrow V \times I \times D^{N}$ and $\mathscr{F}: V \times I \times D^{N} \approx V \times I \times D^{N}$ by $f(x, t) \equiv L_{2}^{-1} H\left(L_{1}(x, t), 0\right)$ and $\mathscr{F}(x, t, y) \equiv L_{2}^{-1} H L_{3}(x, t, y)$. Then $(f, \mathscr{F})$ is $F$-admissible. Furthermore, if we let $F_{1} \equiv p_{1} f(, 0): V \rightarrow V$ and set $F \equiv F_{1} F_{0}$, then $W \approx U_{F}$ and also $F_{1} \simeq$ identity (see the proof of Lemma 9.2(b)). It follows now immediately from the various definitions involved that $\lambda \phi_{1}([f, \mathscr{F}])=\left[F_{1}\right]=\left[F F_{0}^{-1}\right]$.

Conversely, suppose that $W \approx U_{F}$, where $\left[F F_{0}^{-1}\right] \in \operatorname{Im} \lambda \phi_{1} \subseteq \pi_{0} D_{V}^{0}$. Let $[f, \mathscr{F}]$ $\in \theta_{F}^{1}(V)$ be such that $\lambda \phi_{1}([f, \mathscr{F}])=\left[F F_{0}^{-1}\right]^{-1}=\left[F_{0} F^{-1}\right]$, where $\mathscr{F}: V \times I \times D^{N} \rightarrow$ $V \times I \times D^{N}$. Assume $W=U_{F}$ and consider $U \subseteq U_{F}$. Let $L_{1}: V \times I \approx \rightarrow U, L_{1}(x, 0)=x$, be a collar of $V \subseteq U$. Define $L_{2}: V \times I \times D^{N} \rightarrow W \times D^{N}, L_{3}: V \times I \times D^{N} \rightarrow W \times D^{N}$ by $L_{2}(. x, t, y)=\left(L_{1}(x, t), y\right) \quad$ and $\quad L_{3} \equiv L_{2} \mathscr{F}$. Let $\quad M^{k+1^{\prime}} \equiv \mathrm{Cl}\left(W-L_{1}(V \times I)\right) \times 0 \cup$ 
$L_{3}(V \times I \times 0)$. Then $M^{\prime} \approx M$ since $\left(F_{0} F^{-1}\right) F=F_{0}$. Furthermore, we can define $L_{4}: M^{\prime} \times D^{N} \approx W \times D^{N}$ by

$$
L_{4}(x, y)=(x, y), x \in \mathrm{Cl}\left(W-L_{1}(V \times I)\right) \times 0, \quad L_{4}\left(L_{3}(x, t, 0), y\right)=L_{3}(x, t, y) .
$$

$L_{4}$ is well defined because $(f, \mathscr{F})$ is $F$-admissible. But then $L_{4}$ gives us a s.t.h.e. between $M$ and $W$, i.e., $W \in \theta_{T}(M)$.

Corollary 5.1. Let $M^{k+1}$ be as in the theorem. Suppose that $M \approx D U$, and let $W^{k+1} \in \theta(M)$. Then $W \in \theta_{T}(M)$ if and only if $W \approx U_{F}$, where $[F] \in \operatorname{Im} \lambda \phi_{1} \subseteq \pi_{0} D_{V}^{0}$.

Proof. An easy application of Theorem 5 , by setting $F_{0} \equiv$ identity.

Theorem 5 and its corollary show us the connection between the first chapter and stable tangential homotopy equivalences. We have obtained a criterion for when an element of $\theta(M)$ actually lies in $\theta_{T}(M)$. As a special case we get the following:

COROLlary 5.2. With the notation and hypotheses as in Corollary 5.1 assume that $\phi_{1}$ is onto, i.e. $\partial_{1}=0$. Then $\theta(M)=\theta_{T}(M)$.

Proof. Since $\phi_{1}$ is onto, so is $\lambda \phi_{1}$. Now apply Corollary 5.1.

In the light of Corollary 5.2 we must point out a certain incompleteness in Theorem 5. If, in the situation of Corollary 5.1, $\lambda \phi_{1}$ is not onto, it does not follow that $\theta_{T}(M) \neq \theta(M)$. The difficulty arises essentially from Lemma 9.2(c). Let $B$ be the subgroup of $\pi_{0} D_{V}^{0}$ generated by those $[H]$ such that $H$ extends to $U$, and let $p: \pi_{0} D_{V}^{0} \rightarrow \pi_{0} D_{V}^{0} / B$ be the canonical projection on the left coset space. Then it is the case that $p \lambda \phi_{1}$ is onto if and only if $\theta_{T}(M)=\theta(M)$. Unfortunately, it does not seem easy to get hold of $B$ and so Theorem 5 is useful mainly in giving positive results. To obtain results of the type $\theta(M) \neq \theta_{T}(M)$, arguments based on Pontrjagin classes are easier.

Now it happens that the generalized homotopy groups which we have used can sometimes be computed in terms of the usual homotopy groups. This is especially true for the spaces $S^{k} \times S^{l}$ and so, as an example, we conclude with one trivial application of Corollary 5.2 which is proved by some easy computations of homotopy groups.

Corollary 5.3. $\theta\left(S^{4} \times S^{7}\right)=\theta_{T}\left(S^{4} \times S^{7}\right)$.

\section{REFERENCES}

1. M. K. Agoston, On handle decompositions and diffeomorphisms, Trans. Amer. Math. Soc. 137 (1969), 21-26.

2. A. Haefliger, Plongements différentiables de variétés dans variétés, Comment. Math. Helv. 36 (1961), 47-82.

3. - Knotted (4k-1)-spheres in 6k-space, Ann. of Math. 75 (1962), 452-466.

4. - Differentiable embeddings of $S^{n}$ in $S^{n+q}$ for $q>2$, Ann. of Math. 83 (1966), 402-436. 
5. W. C. Hsiang, J. Levine, and R. H. Szczarba, On the normal bundle of a homotopy sphere embedded in Euclidean space, Topology 3 (1965), 173-181.

6. I. M. James, On the iterated suspension, Quart. J. Math. Oxford Ser. 5 (1954), 1-10.

7. M. Kervaire, An interpretation of $G$. Whitehead's generalization of $H$. Hopf's invariant, Ann. of Math. 69 (1959), 345-365.

8. - A manifold which does not admit any differentiable structure, Comment. Math. Helv. 34 (1960), 257-270.

9. M. Kervaire and J. Milnor, Groups of homotopy spheres. I, Ann. of Math. 77 (1963), 504-537.

10. R. Lashoff, Some theorems of Browder and Novikov on homotopy equivalent manifolds with an application, Univ. of Chicago Notes, prepared by R. De Sapio.

11. J. Levine, A classification of differentiable knots, Ann. of Math. 82 (1965), 15-50.

12. J. Milnor, A procedure for killing homotopy groups of differentiable manifolds, Proc. Symps. Pure Math., Vol. 3, Amer. Math. Soc., Providence, R. I., 1961, pp. 39-55.

13. - Lectures on the h-cobordism theorem, Princeton Univ. Press, Princeton, N. J., 1965.

14. S. Smale, On the structure of manifolds, Amer. J. Math. 84 (1962), 387-399.

15. C. T. C. Wall, Classification of (n-1)-connected 2 -manifolds, Ann. of Math. 75 (1962), 163-189.

16. Yuh-lin Jou, Pseudo-manifold and manifold homotopy groups, J. Chinese Math. Soc. (N. S.) 1 (1951), 164-206.

\author{
YALE UNIVERSITY, \\ New Haven, Connecticut \\ WESLEYAN UNIVERSITY, \\ Middletown, Connecticut
}

